\title{
DYNAMIC MODELING AND RESPONSE OF RIGID EMBEDDED CYLINDERS
}

\author{
A.S. Veletsos, A.H. Younan and K. Bandyopadhyay
}

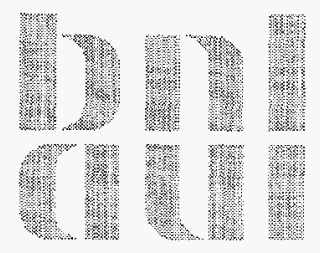

October 1994

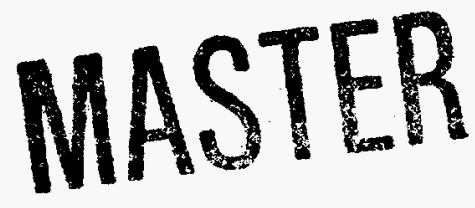

Prepared for

OFFICE OF ENVIRONMENTAL RESTORATION AND WASTE MANAGEMENT

DEPARTMENT OF ENERGY, WASHINGTON, D.C.

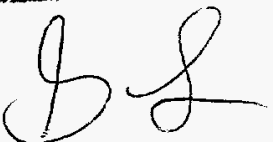


This report was prepared as an account of work sponsored by an agency of the United States Government. Neither the United States Government nor any agency thereof, nor any of their employees, nor any of their contractors, subcontractors, or their employees, makes any warranty, express or implied, or assumes any legal liability or responsibility for the accuracy, completeness, or usefulness of any information, apparatus, product, or process disclosed, or represents that its use would not infringe privately owned rights. Reference herein to any specific commercial product, process, or service by trade name, trademark, manufacturer, or otherwise, does not necessarily constitute or imply its endorsement, recommendation, or favoring by the United States Government or any agency, contractor or subcontractor thereof. The views and opinions of authors expressed herein do not necessarily state or reflect those of the United States Government or any agency, contractor or subcontractor thereof.

Printed in the United States of America Available from

National Technical Information Service

U.S. Department of Commerce

5285 Port Royal Road Springfield, VA 22161

NTIS price codes:

Printed Copy: A04; Microfiche Copy: A01 


\section{DISCLAIMER}

Portions of this document may be illegible in electronic image products. Images are produced from the best available original document. 


\begin{abstract}
Following a brief review of the limitations of a popular technique for modeling the soil action in analyses of the dynamic response of deeply embedded cylindrical foundations and structures, the sources of the limitations are identified and a modification is proposed which, while retaining the attractiveness of the original model, defines correctly the action of the system. In the proposed approach, the soil medium is modeled by a series of elastically constrained, rather than unconstrained, thin horizontal layers with a circular hole at the center. The impedances of the constrained layers are established and are then used to evaluate the dynamic response of a rigid vertical cylinder embedded in a uniform viscoelastic stratum of constant thickness and infinite extent in the horizontal direction. The cylinder and the stratum are presumed to be supported on a non-deformable base undergoing a uniform horizontal motion. The effects of both harmonic and earthquake induced ground motions are considered. The response quantities examined include the dynamic force per unit of cylinder height and the corresponding base shear and base moment.
\end{abstract}





\section{TABLE OF CONTENTS}

Section

Page

ABSTRACT

iii

TABLE OF CONTENTS

$\mathrm{v}$

LIST OF FIGURES

vii

LIST OF TABLES

ix

EXECUTIVE SUMMARY

xi

ACKNOWLEDGMENT

xiii

1 INTRODUCTION

1-1

2 SYSTEM

2-1

3 MODELING OF MEDIUM WITH UNCONSTRAINED LAYERS

3.1 Layer Impedance

3-1

3.2 Response of System

3-2

4 PROPOSED MODELING OF MEDIUM

4-1

4.1 Shearing Resistance of Medium

4-1

4.2 Layer Impedance

4-3

4.3 Response of System

4-4

4.4 Relation to Previous Studies

4-5

5 RESPONSE DATA FOR CYLINDER-SOIL SYSTEM

5.1 Base Shear

$5-1$

5.2 Relative Contributions of Normal and Shearing Stresses

$5-2$

5.3 Distribution of Cylinder Forces and Base Moment

$5-3$

5.4 A Final Comment

5-3

6 CONCLUSIONS

6-1

7 REFERENCES

7-1

8 APPENDIX

8-1

9 NOTATION

9-1 


\section{LIST OF FIGURES}

Figure

Page

2.1 System Considered.

3.1 Impedance Coefficients for Baranov-Novak Layers with $\delta=0$ : (a) Plane Strain Condition; and (b) Plane Stress Condition.

3.2 Modeling of System with Baranov-Novak Layers: (a) Plan View; and (b) Elevation View.

4.1 Effect of Horizontal Shearing Resistance of Medium: (a) Horizontal Shearing Stresses for an Element; and (b) Elastically Constrained Layer in Proposed Modeling of Medium.

4.2 Impedance Factors $\tilde{\alpha}_{\mathrm{n}}$ and $\tilde{\beta}_{\mathrm{n}}$ for Elastically Constrained Layers; $v=1 / 3, \delta=0$.

4.3 Impedance Factors $\alpha_{\mathrm{n}}$ and $\beta_{\mathrm{n}}$ for Elastically Constrained Layers; $v=1 / 3, \delta=0$.

5.1 Amplification Factors for Base Shear in Cylinder of Harmonically Excited Systems; $v=1 / 3, \delta=0.1$.

5.2 Amplification Factors for Base Shear in Cylinder of Systems Subjected to El Centro Earthquake Record; $v=1 / 3, \delta=0.1$.

5.3 Effect of Stratum-Height to Cylinder-Radius Ratio on Absolute Maximum Amplification Factor for Base Shear in Cylinder of Base-Excited Systems; $v=1 / 3, \delta=0.1$.

5.4 Real-Valued Amplitude of Layer Impedance Contributed by Normal Pressures; $v=1 / 3, \delta=0.1$. 


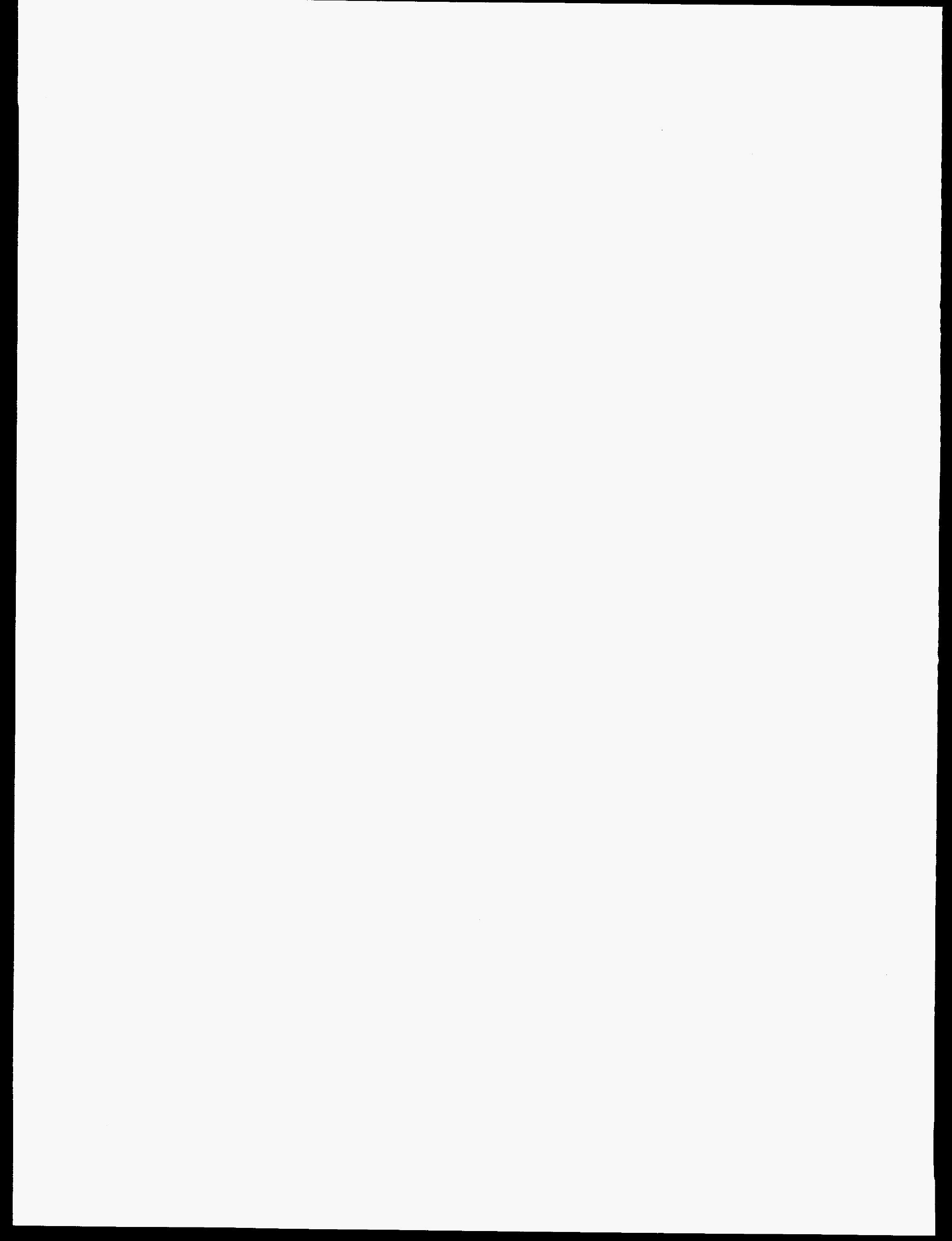




\section{LIST OF TABLES}

Table

Page

4.1 Values of Dimensionless Coefficient $C_{n}$ in Expression of $\left(K_{n}\right)_{s t}$.

5.1 Static Values of Base Shear and Base Moment in Systems with Different $\mathrm{H} / \mathrm{R}$ ratios. 


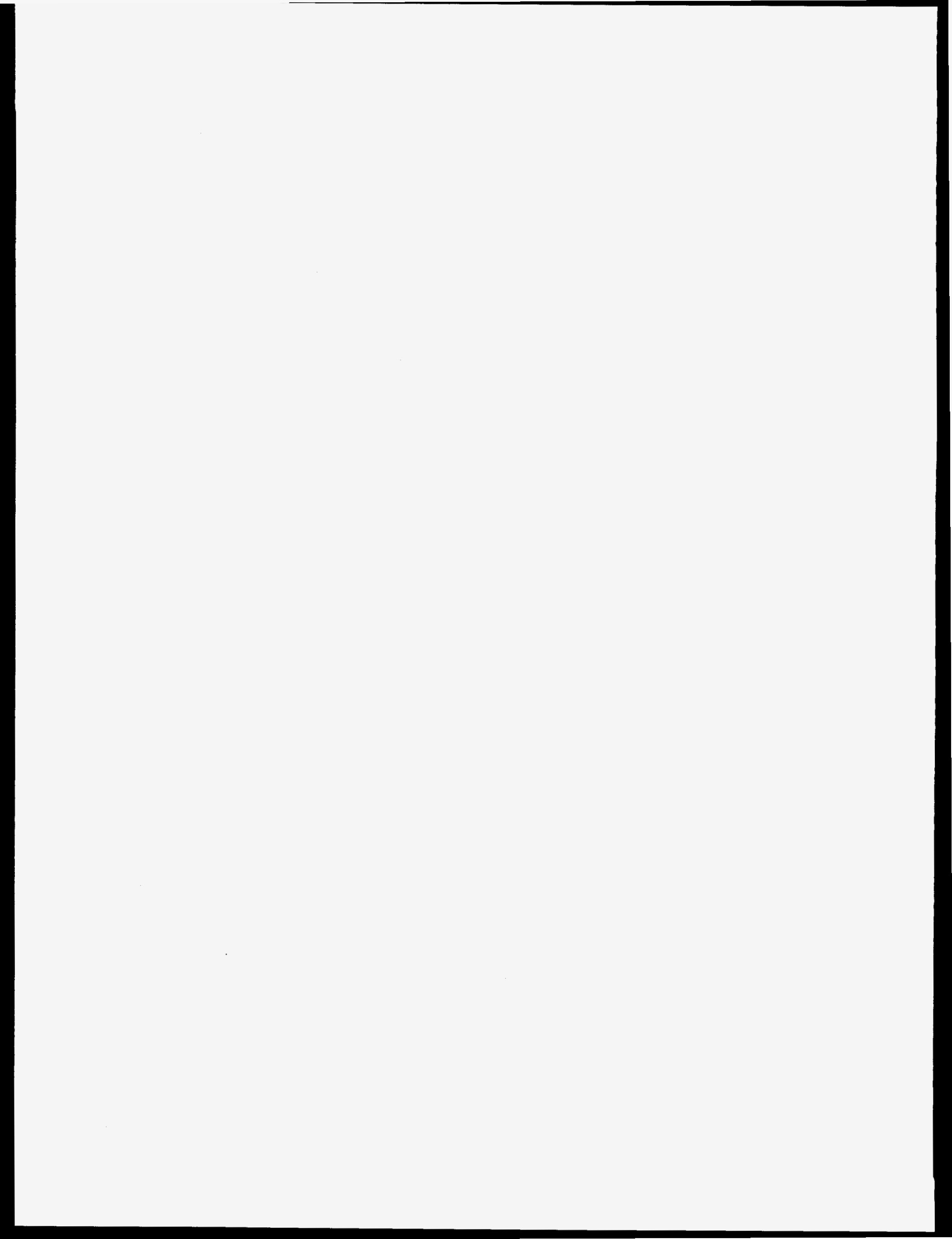




\section{EXECUTIVE SUMMARY}

The study reported herein is the fourth in a series of investigations of the response to ground shaking of retaining walls and deeply embedded vertical cylindrical structures. The objectives of these studies have been to provide improved insights into the dynamic responses of these systems and to formulate rational but simple methods for their analysis and design. The previous studies were described in Brookhaven National Laboratory reports 52357, 52372 and 52402.

Probably the simplest available method for approximating the soil action in analyses of the dynamic response of cylindrical foundations and embedded structures is the one based on the Baranov-Novak idealization of the medium. In this approach, the soil is modeled by a series of independent thin layers with a circular hole at the center and their resistance to deformation is expressed by their impedance, defined as the complex-valued amplitude of a harmonic force which when applied along the inner circular boundary in the direction of the desired response will induce a steady-state displacement of unit amplitude in that direction.

Following a brief review of the limitations of this popular technique for modeling the action of the soil medium, the sources of the limitations are identified and a modification is proposed which, while retaining the attractiveness of the original model, defines correctly the action of the system. In the proposed approach, the soil medium is modeled by a series of elastically constrained, rather than unconstrained, thin horizontal layers with a circular hole at the center. The impedances of the constrained layers are established and then used to evaluate the dynamic response of a rigid vertical cylinder embedded in a uniform viscoelastic stratum of constant thickness and infinite extent in the horizontal direction. The cylinder and the stratum are presumed to be supported on a non-deformable base undergoing a uniform horizontal motion. The effects of both harmonic and earthquake induced ground motions are considered. The response quantities examined include the dynamic force per unit of cylinder height and the corresponding base shear and base moment.

The principal conclusions of the study may be summarized as follows:

1. In analyses of the dynamic response of deeply embedded vertical cylindrical structures, the representation of the soil medium by a series of independent Baranov-Novak layers may lead to major inaccuracies, particularly for broad structures with ratios of stratum-height to cylinder-radius of 2 or less. These inaccuracies stem from the failure of this representation to provide for the capacity 
of the medium between the structure and the far field to transfer forces vertically by horizontal shearing action. The greater the horizontal shearing stiffness of the medium relative to its corresponding extensional stiffness, the greater are generally the resulting discrepancies. This deficiency in modeling may be eliminated by considering the individual layers to be elastically supported to a common base.

2. In addition to a dimensionless measure of the frequency of the excitation, Poisson's ratio for the medium, and the associated material damping factor, the impedance of the elastically constrained layer depends on the stiffness of the supporting springs. The latter stiffness, in turn, depends on the height-to-radius ratio of the embedded cylinder and on the order of the natural mode in which the soil stratum is vibrating. With the impedances of the elastically constrained layer established, the pressures on and forces in the cylinder for harmonically excited systems may be determined from the expressions presented herein, and those for systems subjected to transient excitations may be evaluated by Fourier transform techniques.

3. The data presented herein are limited to rigid vertical cylinders and to a non-deformable, moving base. The presence of a flexible rather than rigid cylinder will reduce the effective horizontal extensional stiffness of the medium relative to its shearing stiffness. This reduction, in turn, will increase the forces that get transmitted to the base by horizontal shearing action, and hence the differences between the solutions obtained with the conventional, unconstrained layers and the proposed, constrained layers. By contrast, the presence of a flexible rather than rigid supporting medium will have the opposite effects. These effects will be examined in future publications. 


\section{ACKNOWLEDGMENT}

This study was carried out at Rice University in cooperation with Brookhaven National Laboratory (BNL). The authors are grateful to the Department of Energy Program Directors John Tseng, James Antizzo and Dinesh Gupta for supporting the study, and to Morris Reich of BNL for his encouragement and support. 


\section{SECTION 1}

\section{INTRODUCTION}

Probably the simplest available method for approximating the soil action in analyses of the dynamic response of cylindrical foundations and embedded structures is the one based on the Baranov-Novak (B-N) idealization of the medium [Baranov (1967), Beredugo and Novak (1972), Novak (1974)]. In this approach, the soil is modeled by a series of independent thin layers with a circular hole at the center, and their resistance to deformation is expressed by their impedance, defined as the complexvalued amplitude of a harmonic force which when applied along the inner circular boundary in the direction of the desired response will induce a steady-state displacement of unit amplitude in that direction.

The B-N layers have been used extensively in analyses of the dynamic response of piles [e.g., Novak (1974), Veletsos and Dotson (1988)], cylindrical foundations [e.g., Novak and Beredugo (1972), Beredugo and Novak (1972), and Novak and Sachs (1973)], and deeply embedded structures [Miller et al. (1992)]. With a simpler, real-valued definition of the layer impedance, a similar model has also been used by Scott (1973) and by others [Karkanias (1983), Dennehy (1984), Jain and Scott (1989), Alampali and Elgamal (1991)] in dynamic analyses of straight retaining walls, which represent the limiting form of a cylinder with a radius tending to infinity.

The representation of the soil medium by B-N layers has been shown to be quite accurate for the analysis of piles [Nogami and Novak (1977)] which are rather slender structural elements with large height-to-radius ratios. By contrast, Scott's approach to the study of straight retaining walls has been shown to lead to major inaccuracies [Veletsos and Younan (1994c)]. This difference clearly suggests that the appropriateness of the B-N layers depends importantly on the height-to-radius ratio of the structure being investigated.

The objectives of this paper are: (1) to assess the reliability of the B-N layer modeling in analyses of the response to ground shaking of a rigid vertical cylinder embedded in a viscoelastic stratum; (2) to identify the source or sources of any possible deficiencies; and (3) if necessary, to propose a modification, which while retaining the attractiveness of the original model, eliminates its deficiencies. The response quantities examined include the dynamic force per unit of cylinder height and the corresponding base shear and base moment. The effects of both harmonic and earthquakeinduced ground motions are considered. 


\section{SECTION 2}

\section{SYSTEM}

The system investigated is shown in Fig. 2-1. It is a rigid, vertical cylinder of radius $R$ that is embedded in a uniform viscoelastic stratum of thickness $\mathrm{H}$ and infinite extent in the horizontal plane. The stratum is free at its upper surface and, along with the cylinder, is supported on a non-deformable rigid base undergoing a horizontal motion the acceleration of which at any time $t$ is $\ddot{x}_{g}(t)$. Complete bonding is assumed at the interfaces of the cylinder, medium and their base. Material damping for the medium is presumed to be of the constant hysteretic type. Points in the medium are defined by the cylindrical coordinate system $r, \theta, z$, the origin of which is taken at the center of the cylinder-base, with $\theta$ positive in the counter-clockwise direction and $\theta=0$ coinciding with the positive direction of the ground motion.

The properties of the medium are defined by its mass density $\rho$, shear modulus $G$, Poisson's ratio $v$, and the material damping factor $\delta$, which is considered to be frequency-independent and the same for both shearing and axial deformations. The latter factor is the same as the tan $\delta$ factor used by the senior author and his associates in studies of foundation dynamics and soil-structure interaction [e.g., Veletsos and Verbic (1973), Veletsos and Nair (1975), Veletsos and Dotson (1988)], and twice as large as the percentage of critical damping $\beta$ which has been used by other authors in related studies [e.g., Tajimi (1969), Roesset et al. (1973), Tassoulas and Kausel (1983), Pais and Kausel (1988)]. 

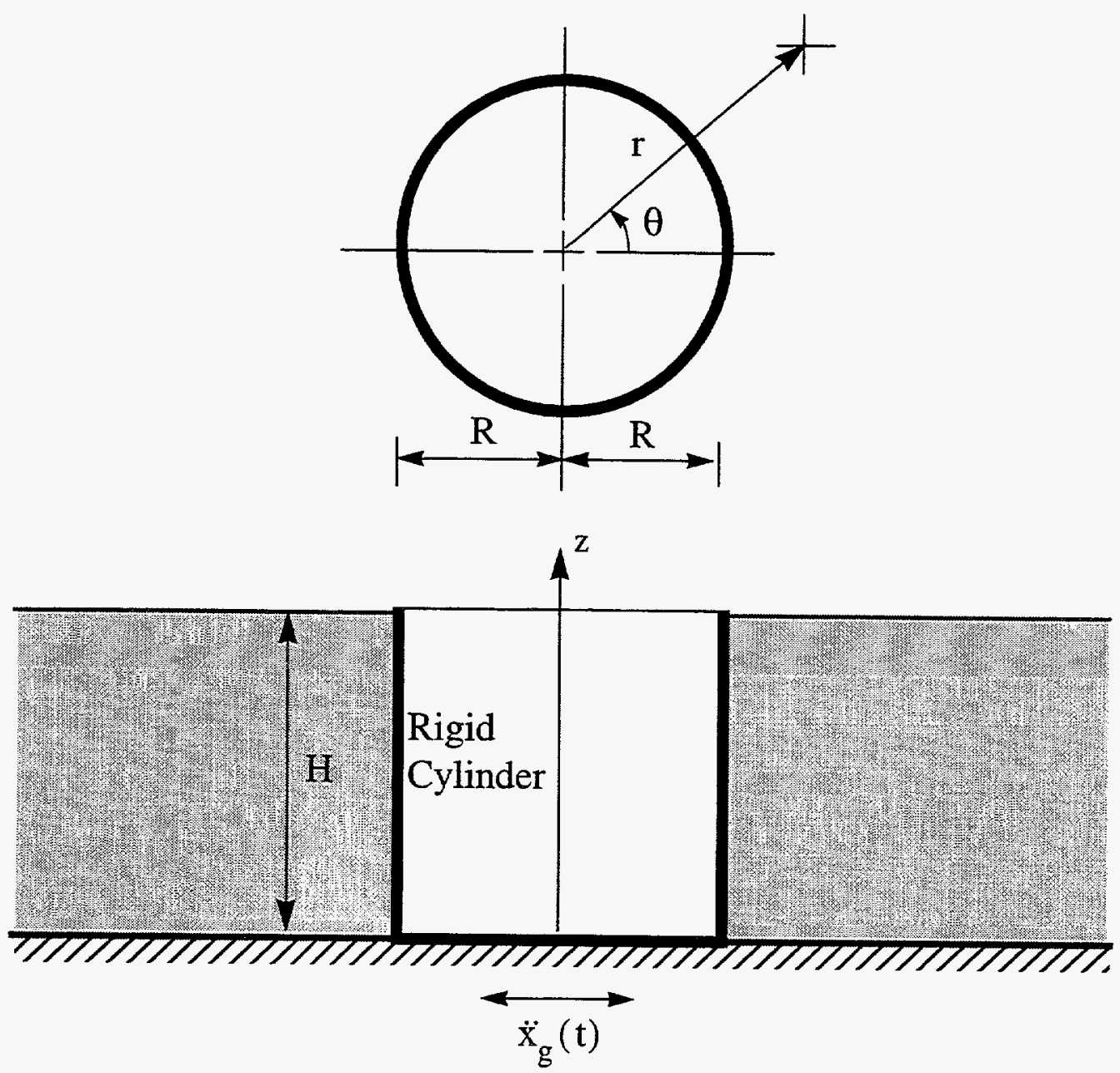

Fig. 2-1 System Considered 


\section{SECTION 3}

\section{MODELING OF MEDIUM WITH UNCONSTRAINED LAYERS}

In the $\mathrm{B}-\mathrm{N}$ idealization of the medium, the stratum is represented by a set of independent, unconstrained layers with a non-deformable circular hole of radius $\mathrm{R}$ at the center. The individual layers are presumed to be of unit thickness and in a state of plane strain, i.e., to experience no vertical strains.

\subsection{Layer Impedance}

The horizontal impedance of a layer, $\mathrm{K}$, may conveniently be expressed in the form suggested by Veletsos and Dotson (1988) as

$$
\mathrm{K}=1.5 \pi \mathrm{G}\left(\alpha+\mathrm{ia}{ }_{0} \beta\right)
$$

where $\mathrm{i}=\sqrt{-1} ; \mathrm{a}_{0}$ is a dimensionless frequency parameter defined by

$$
a_{0}=\frac{\omega R}{v_{s}}
$$

$\omega=$ the circular frequency of the exciting force and of the resulting steady-state response; $v_{s}=\sqrt{G / p}=$ the shear wave velocity for the medium; and $\alpha$ and $\beta$ are dimensionless factors that depend on $a_{0}$, Poisson's ratio for the medium $v$, and the associated material damping factor $\delta$. The real part of Eq. (1) is equivalent to the effect of a linear spring, whereas the imaginary part is equivalent to that of a viscous damper arranged in parallel with the spring. The factors $\alpha$ and $\beta$ will be referred to in the following as the stiffness and damping coefficients, respectively. Fig. 3-1(a) shows the variations of $\alpha$ and $\beta$ as a function of $a_{0}$ for undamped layers with several different values of $v$ in the range between zero and 0.5 . For $v=1 / 3$, as $a_{0} \rightarrow \infty, \alpha \rightarrow 1$ and $\beta \rightarrow 2$.

If instead of a plane strain condition, the layer were presumed to be in a state of plane stress (i.e., to be free of any vertical stresses), the variations of $\alpha$ and $\beta$ would be as shown in Fig. 3-1(b). Note that these results are significantly less sensitive to changes in $v$ than are those obtained with the plane strain assumption. The plane strain condition represents accurately the state of stresses around long, slender structural elements such as piles, but for the relatively broad cylinders that are of primary concern in this study, the plane stress assumption has been shown to be superior [Veletsos and Younan 
(1994a), (1994b)], and unless specifically otherwise indicated, the layers in the following developments will be presumed to be in a state of plane stress.

\subsection{Response of System}

Under the influence of the ground motion, a thin cylindrical element of the medium at $r \rightarrow \infty$ will respond as a uniform, vertical cantilever shear-beam. The stratum may then be modeled by a shearbeam at the far-field and a series of B-N layers between the shear-beam and the cylinder, as shown in Fig. 3-2.

Let $u_{f}(\eta, t)$ be the instantaneous value of the horizontal displacement relative to the moving base of a point on the shear-beam at a dimensionless distance $\eta=\mathrm{z} / \mathrm{H}$ from the base. For a harmonic base motion of acceleration

$$
\ddot{x}_{g}(t)=\ddot{X}_{g} e^{i \omega t}
$$

where $\ddot{\mathrm{X}}_{\mathrm{g}}=$ the acceleration amplitude,

$$
\mathfrak{u}_{f}(\eta, t)=U_{f}(\eta) e^{i \omega t}
$$

and the displacement amplitude $U_{f}(\eta)$ may be expressed by modal superposition as

$$
U_{f}(\eta)=\sum_{n=1}^{\infty} U_{n} \sin \left[\frac{(2 n-1) \pi}{2} \eta\right]
$$

where

$$
\begin{aligned}
& U_{n}=-\frac{16}{\pi^{3}} \frac{\rho \ddot{X}_{g} H^{2}}{G} \frac{1}{(2 n-1)^{3}} \frac{1}{1-\phi_{n}^{2}+i \delta} \\
& \phi_{n}=\frac{\omega}{\omega_{n}}
\end{aligned}
$$

and $\omega_{n}=$ the nth circular natural frequency of the shear-beam, given by

$$
\omega_{\mathrm{n}}=\frac{(2 \mathrm{n}-1) \pi}{2} \frac{\mathrm{v}_{\mathrm{s}}}{\mathrm{H}}
$$

The instantaneous value of the resulting horizontal dynamic force per unit of cylinder height, $F(\eta, t)$, may then be expressed as the product of the layer impedance $K$ and the difference in the displacements of the shear-beam at the far field and of the cylinder at the center. Furthermore, since 
the motion of the cylinder is the same as that of the base, this force may also be expressed in terms of the relative displacement of the shear-beam $u_{f}(\eta, t)$, as

$$
F(\eta, t)=K u_{f}(\eta, t)=K U_{f}(\eta) e^{i \omega t}
$$

As far as can be determined, this expression was first used by Scott (1973) in a study of the dynamic response of straight retaining walls, and its rationale was further examined by Veletsos and Younan (1994c).

With the dynamic force per unit of cylinder height determined, the instantaneous values of the total dynamic force or base shear in the cylinder, $Q_{b}(t)$, and the associated overturning base moment, $M_{b}(t)$, are determined by integration to be

$$
\mathrm{Q}_{\mathrm{b}}(\mathrm{t})=\int_{0}^{1} \mathrm{~F}(\eta, \mathrm{t}) \mathrm{Hd} \eta=\frac{2}{\pi} \mathrm{KH} \sum_{n=1}^{\infty} \frac{1}{2 n-1} \mathrm{U}_{\mathrm{n}} \mathrm{e}^{\mathrm{i} \omega \mathrm{t}}
$$

and

$$
M_{b}(t)=\int_{0}^{1} F(\eta, t) H^{2} \eta d \eta=\frac{4}{\pi^{2}} K H^{2} \sum_{n=1}^{\infty} \frac{(-1)^{n-1}}{(2 n-1)^{2}} U_{n} e^{i \omega t}
$$

Finally, with the harmonic response of the system established, the response to a transient excitation may be determined by Fourier transform techniques. Being a function of the frequency of the motion, the impedance of the layers $\mathrm{K}$ in the latter case must be evaluated for each harmonic component of the forcing function. Naturally, if $\mathrm{K}$ were or could be considered to be frequency-independent, the force $F(\eta, t)$ for an arbitrary transient excitation and the associated base shear and base moment could be evaluated from Eq. (9) merely as the product of $\mathrm{K}$ and the transient response of the shear-beam at the far field. This approximation was used in the studies reported both by Scott (1973) and by Miller et al. (1992), although the value of K in Scott's study differed significantly.

Referring back to Fig. 3-1, it is worth noting that as $a_{0} \rightarrow 0$ the stiffness coefficient $\alpha$, and hence the dynamic base shear and base moment for the cylinder, also tend to zero, a result that is clearly unacceptable. This deficiency is normally overcome by taking as the low-frequency limit of $\alpha$ the value corresponding to some small but finite value of $a_{0}$. 







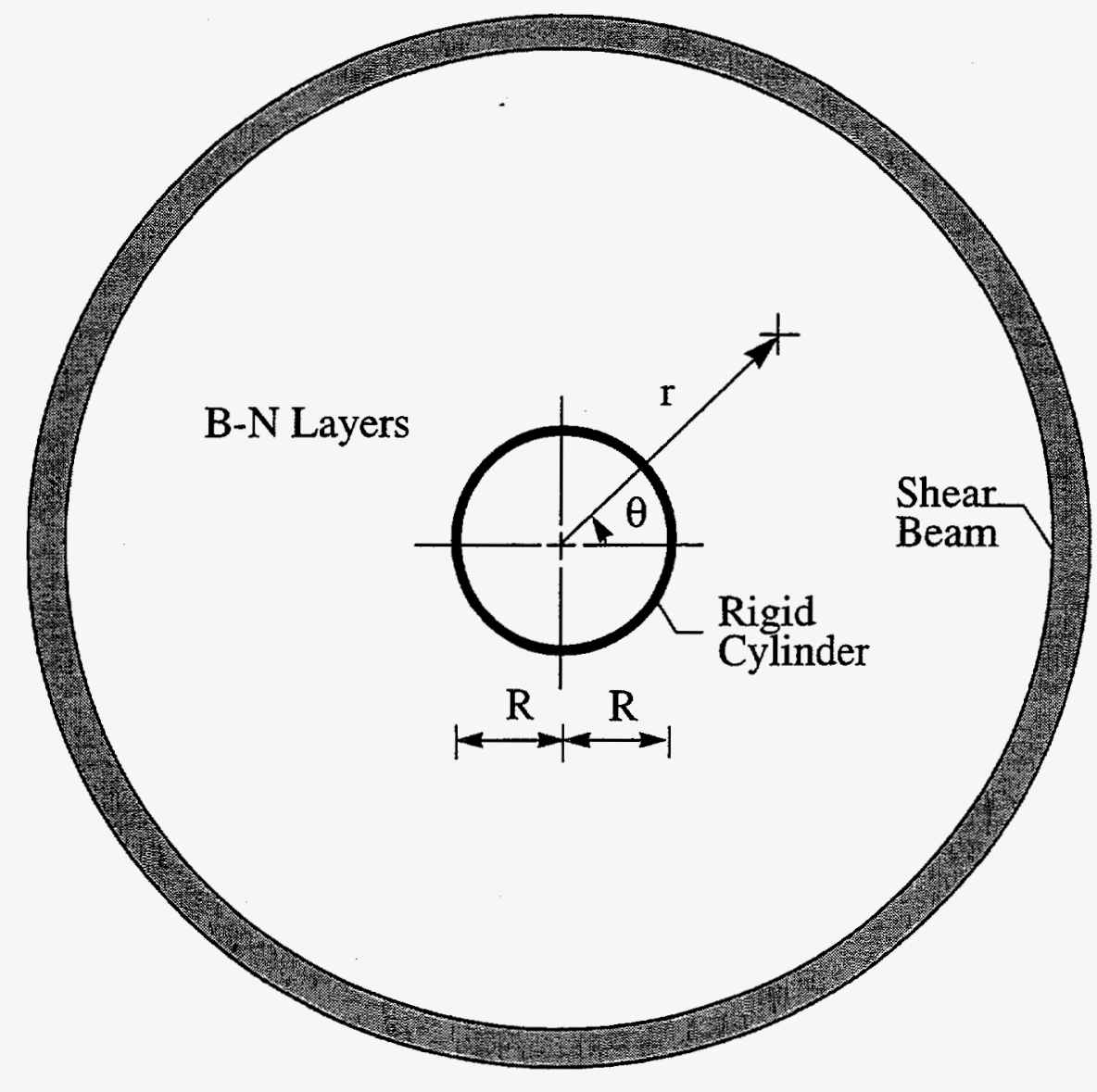

(a)

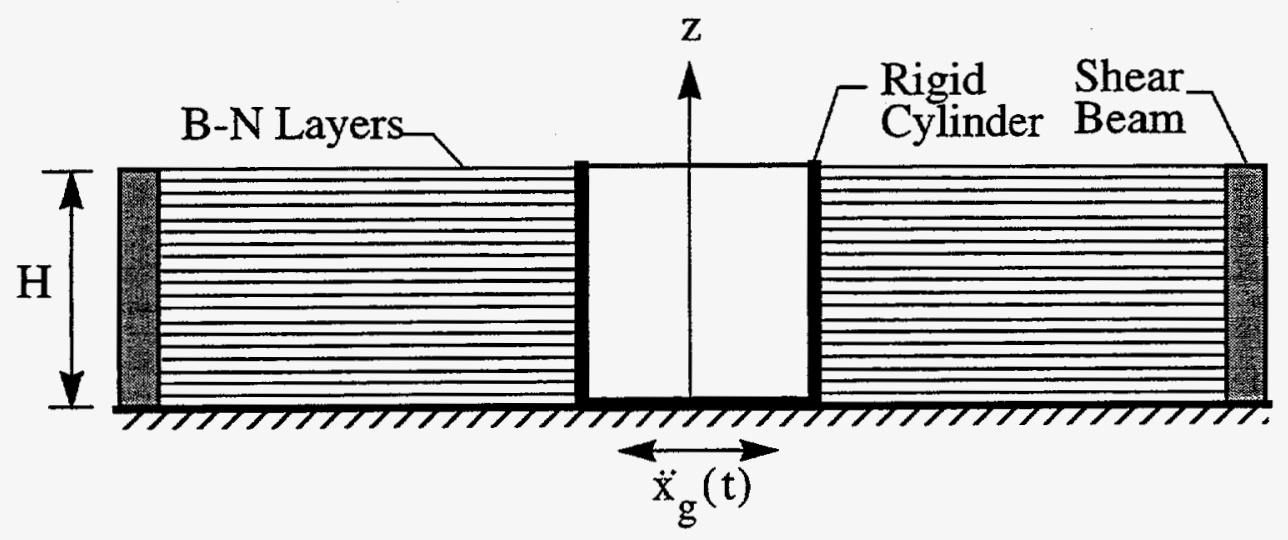

(b)

Fig. 3-2 Modeling of System with Baranov-Novak Layers: (a) Plan View; and (b) Elevation View. 


\section{SECTION 4}

\section{PROPOSED MODELING OF MEDIUM}

Apart from its failure to predict the response of the system for values of $a_{0} \rightarrow 0$, the model referred to in the preceding section does not provide for the capacity of the medium between the far field and the cylinder to transfer forces vertically by horizontal shearing action.

\subsection{Shearing Resistance of Medium}

In addition to the horizontal normal stresses and inertia forces, an element of the medium defined by the infinitesimal coordinate increments $d r, d \theta$ and $d z$ is acted upon at its upper and lower faces by radial and circumferential shearing stresses $\tau_{z \mathrm{r}}$ and $\tau_{z \theta}$, as shown in Fig. 4-1(a). On the assumption that the horizontal variation of the vertical displacement of the medium $w$ is negligible, a quite reasonable assumption for the horizontal base motion examined herein, the horizontal components of the shearing stresses can be expressed as

$$
\begin{aligned}
\tau_{z r} & =\frac{G}{H} \frac{\partial u}{\partial \eta} \\
\tau_{z \theta} & =\frac{G}{H} \frac{\partial v}{\partial \eta}
\end{aligned}
$$

where $\mathrm{u}$ and $\mathrm{v}$ are the radial and circumferential displacement components of the medium relative to the moving base. The differences in the shearing stresses acting on the top and bottom faces of the element are then given by

$$
\begin{aligned}
& \Delta \tau_{\mathrm{zr}}=\frac{\partial \tau_{\mathrm{zr}}}{\partial \mathrm{z}}=\frac{\mathrm{G}}{\mathrm{H}^{2}} \frac{\partial^{2} \mathrm{u}}{\partial \eta^{2}} \\
& \Delta \tau_{\mathrm{z} \theta}=\frac{\partial \tau_{\mathrm{z} \theta}}{\partial \mathrm{z}}=\frac{\mathrm{G}}{\mathrm{H}^{2}} \frac{\partial^{2} \mathrm{v}}{\partial \eta^{2}}
\end{aligned}
$$

The displacements $u$ and $v$ can now be expressed by the method of separation of variables as linear combinations of the natural modes of vibration of the stratum considered to act as a cantilever shearbeam, in the form 


$$
\mathrm{u}(\xi, \eta, \theta, \mathrm{t})=\sum_{\mathrm{n}=1}^{\infty} \mathrm{g}_{\mathrm{n}}(\xi, \theta, \mathrm{t}) \sin \left[\frac{(\mathrm{n}-1) \pi}{2} \eta\right]
$$

and

$$
v(\xi, \eta, \theta, t)=\sum_{n=1}^{\infty} h_{n}(\xi, \theta, t) \sin \left[\frac{(n-1) \pi}{2} \eta\right]
$$

where $g_{n}$ and $h_{n}$ are functions of the normalized radial position coordinate $\xi=r / H$, and of $\theta$ and $t$. On substituting Eqs. (16) and (17) into Eqs. (14) and (15), the difference in the shearing stresses for the nth natural mode of vibration can be written as

$$
\begin{aligned}
& \left(\Delta \tau_{\mathrm{zr}}\right)_{\mathrm{n}}=-\left[\frac{(2 \mathrm{n}-1) \pi}{2}\right]^{2} \frac{\mathrm{G}}{\mathrm{H}^{2}} \mathrm{u}_{\mathrm{n}} \\
& \left(\Delta \tau_{\mathrm{z} \theta}\right)_{\mathrm{n}}=-\left[\frac{(2 \mathrm{n}-1) \pi}{2}\right]^{2} \frac{\mathrm{G}}{\mathrm{H}^{2}} \mathrm{v}_{\mathrm{n}}
\end{aligned}
$$

The multipliers of $u_{n}$ and $v_{n}$ in the latter expressions are now recognized to equal $\rho \omega_{n}^{2}$; accordingly, Eqs. (18a) and (18b) can also be written as

$$
\begin{aligned}
& \left(\Delta \tau_{\mathrm{zr}}\right)_{\mathrm{n}}=-\rho \omega_{\mathrm{n}}^{2} \mathrm{u}_{\mathrm{n}} \\
& \left(\Delta \tau_{\mathrm{z} \theta}\right)_{\mathrm{n}}=-\rho \omega_{\mathrm{n}}^{2} \mathrm{v}_{\mathrm{n}}
\end{aligned}
$$

Incidentally, the latter expressions could also have been obtained from the dynamic equilibrium of the indicated shearing stresses and the inertial forces acting on the element.

Eqs. (18) and (19) represent forces per unit of area that are proportional and opposite to the modal displacement components $u_{n}$ and $v_{n}$, the constant of proportionality being independent of the position coordinate and a function only of the modal order $\mathbf{n}$ of the response component being considered. Therefore, they may be thought of as being due to massless linear springs of stiffness

$$
k_{n}=\rho \omega_{n}^{2}=\left[\frac{(2 n-1) \pi}{2}\right]^{2} \frac{G}{H^{2}}
$$

that are uniformly distributed in both the radial and circumferential directions. It follows that, for each modal component of response, the horizontal shearing action of the medium may be modeled by such springs, and the medium itself may be modeled by a series of elastically supported layers as shown in Fig. 4-1(b). The lower ends of the springs are presumed to be attached to a common base.

The spring stiffness defined by Eq. (20) is for an undamped medium. The effect of material damping for the medium may be accounted for by replacing its shear modulus $G$ by its complex valued 
modulus

$$
\mathrm{G}^{*}=\mathrm{G}(1+\mathrm{i} \delta)
$$

leading to an effective spring stiffness

$$
k_{n}^{*}=k_{n}(1+i \delta)
$$

\subsection{Layer Impedance}

Let $\mathrm{K}_{\mathrm{n}}$ be the impedance of the elastically supported layer when the stratum is vibrating in its nth natural mode. Derived in the Appendix, $K_{n}$ may be expressed in a form analogous to (1) as

$$
\mathrm{K}_{\mathrm{n}}=1.5 \pi \mathrm{G}\left(\tilde{\alpha}_{\mathrm{n}}+\mathrm{ia}_{\mathrm{o}} \tilde{\beta}_{\mathrm{n}}\right)
$$

where the dimensionless factors $\tilde{\alpha}_{n}$ and $\tilde{\beta}_{n}$ are now functions not only of $a_{o}, v$ and $\delta$, but also of the dimensionless measure of the stiffness of the supporting springs

$$
s_{n}=\sqrt{\frac{k_{n} R^{2}}{G}}
$$

which, on making use of Eq. (20), can also be written as

$$
s_{n}=(2 n-1) \frac{\pi}{2} \frac{R}{H}
$$

Note that $s_{n}$ depends both on the ratio of stratum-height to cylinder-radius, $H / R$, and on the order $n$ of the mode of vibration of the stratum being examined.

The variations of $\tilde{\alpha}_{n}$ and $\tilde{\beta}_{n}$ as a function of the frequency parameter $a_{0}$ are shown in Fig. 4-2 for an undamped layer with $v=1 / 3$. Four values of $s_{n}$ in the range between zero and $\pi$ are considered which, for $n=1$, correspond to the $H / R$ values shown in parentheses. As would be expected, the results for $s_{n}=0$ are identical to those presented in Fig. 3-1(b) for the unconstrained B-N layer, but those for the finite values are substantially different, particularly for the smaller values of $a_{0}$ and $H / R$. The very-low-frequency, static value of $\mathrm{K}_{n}$, identified in the following with the subscripts st, may conveniently be expressed as

$$
\left(K_{n}\right)_{s t}=C_{n} G
$$

where $C_{n}$ is a dimensionless function of $s_{n}$ and $v$, the values of which are listed in Table 4-1. It may be noted that as $s_{n} \rightarrow \infty, C_{n}$ tends to $\pi\left(1+\psi_{0}\right) s_{n}$, where $\psi_{0}$ is a dimensionless function of Poisson's ratio $v$ defined by Eq. (45) in the Appendix.

For the values of $a_{0}$ corresponding to the natural frequencies of the stratum defined by Eq. (8), the stiffness factors $\tilde{\alpha}_{n}=0$, and for the smaller values of $a_{0}$, the damping factors $\tilde{\beta}_{n}=0$. The latter 
result is consistent with the well established fact that the stratum possesses no radiational damping capacity within this frequency range. For values of $a_{0}$ greater than those corresponding to the natural frequencies of the stratum, both the stiffness and damping factors for the elastically constrained layer are in close agreement with those obtained for the unconstrained layer. It follows that the conventional modeling of the stratum by unconstrained B-N layers would be sufficiently accurate in this case, but this clearly would not be true for the lower values of $\mathrm{a}_{0}$.

It is instructive at this stage to express Eq. (23) in terms of the static stiffness $\left(\mathrm{K}_{n}\right)_{s t}$ and all frequency-dependent quantities in this equation in terms of the frequency ratio defined by Eq. (7), which is related to the frequency parameter $a_{0}$ by

$$
\phi_{\mathrm{n}}=\frac{1}{\mathrm{~s}_{\mathrm{n}}} \mathrm{a}_{\mathrm{o}}
$$

This leads to

$$
K_{n}=\left(K_{n}\right)_{s t}\left(\alpha_{n}+i \phi_{n} \beta_{n}\right)
$$

where $\alpha_{n}$ and $\beta_{n}$ are modified dimensionless stiffness and damping factors which, for a given frequency $\omega$, are related to $\tilde{\alpha}_{n}$ and $\tilde{\beta}_{n}$ by

$$
\begin{aligned}
& \alpha_{\mathrm{n}}=\frac{1.5 \pi}{\mathrm{C}_{\mathrm{n}}} \tilde{\alpha}_{\mathrm{n}} \\
& \beta_{\mathrm{n}}=\frac{1.5 \pi \mathrm{s}_{\mathrm{n}}}{\mathrm{C}_{\mathrm{n}}} \tilde{\beta}_{\mathrm{n}}
\end{aligned}
$$

The variations of $\alpha_{n}$ and $\beta_{n}$ with $\phi_{n}$ are shown in Fig. 4-3 for an undamped medium with $v=1 / 3$ and five values of the dimensionless stiffness parameter for the supporting springs $s_{n}$. It is observed that, for $\phi_{n} \leq 1$, the stiffness coefficients $\alpha_{n}$ are practically independent of $s_{n}$ and the damping coefficients $\beta_{n}$ are zero, whereas for $\phi_{n}>1$, both coefficients are quite sensitive to variations in $s_{n}$.

\subsection{Response of System}

With the modal impedances of the elastically constrained layer $K_{n}$ established, the dynamic force per unit of cylinder height and the associated base shear and overturning base moment for the harmonically excited system may be determined, in the spirit of Eqs. (9), (10) and (11), from

$$
F(\eta, t)=\sum_{n=1}^{\infty} K_{n} U_{n} \sin \left[\frac{(2 n-1) \pi}{2} \eta\right] e^{i \omega t}
$$




$$
\begin{aligned}
& Q_{b}(t)=\frac{2 H}{\pi} \sum_{n=1}^{\infty} \frac{1}{2 n-1} K_{n} U_{n} e^{i \omega t} \\
& M_{b}(t)=\frac{4 H^{2}}{\pi^{2}} \sum_{n=1}^{\infty} \frac{(-1)^{n-1}}{(2 n-1)^{2}} K_{n} U_{n} e^{i \omega t}
\end{aligned}
$$

Note that, unlike Eqs. (9) through (11), in which the impedance $\mathrm{K}$ appears as a common multiplier, $K_{n}$ in Eqs. (30) through (32) appears under the summations and has a different value for each $n$.

The response of the system to a transient excitation may be evaluated by Fourier transform techniques in a manner analogous to that described for systems with the conventional, unconstrained B-N layers.

\subsection{Relation to Previous Studies}

The assumptions underlying the proposed analysis of the system with the elastically constrained layers are the same as those underlying the analysis employed by Arias et al. (1981) for the limiting case of a medium retained by a straight wall. The results of the two approaches would, therefore, be identical, and based on the comparative studies reported by Veletsos and Younan (1994c) for the straight wall, they are expected to be of high accuracy. The results would also be identical to those which would be obtained from the analysis presented by Veletsos and Younan (1994b) if, in addition to assuming the absence of any vertical normal stresses, the variation of the vertical displacements in the expressions for the horizontal shearing stresses, $\tau_{\mathrm{zr}}$ and $\tau_{\mathrm{z} \theta}$, was neglected. Finally, they would be identical to those that would be obtained from the method employed by Tajimi (1969) if, in addition to assuming the absence of any vertical displacements, the compressional wave velocity for the medium

$$
v_{c}=\sqrt{\frac{2(1-v)}{1-2 v}} v_{s}
$$

were replaced, in the spirit of a suggestion made by Gazetas and Dobry (1987) for related problems, by

$$
\mathrm{v}_{\mathrm{c}}^{\prime}=\sqrt{\frac{2}{1-v}} \mathrm{v}_{\mathrm{s}}
$$




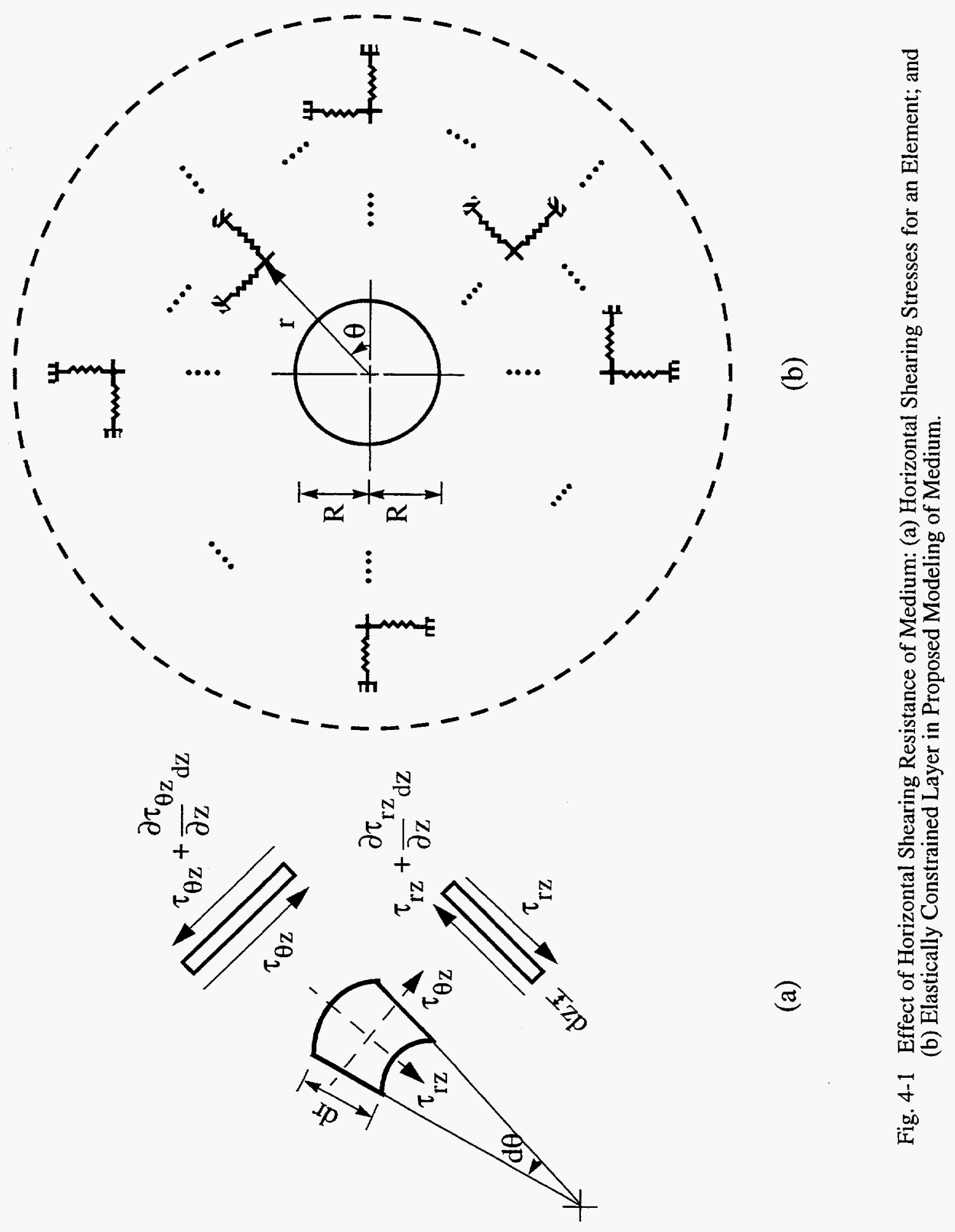




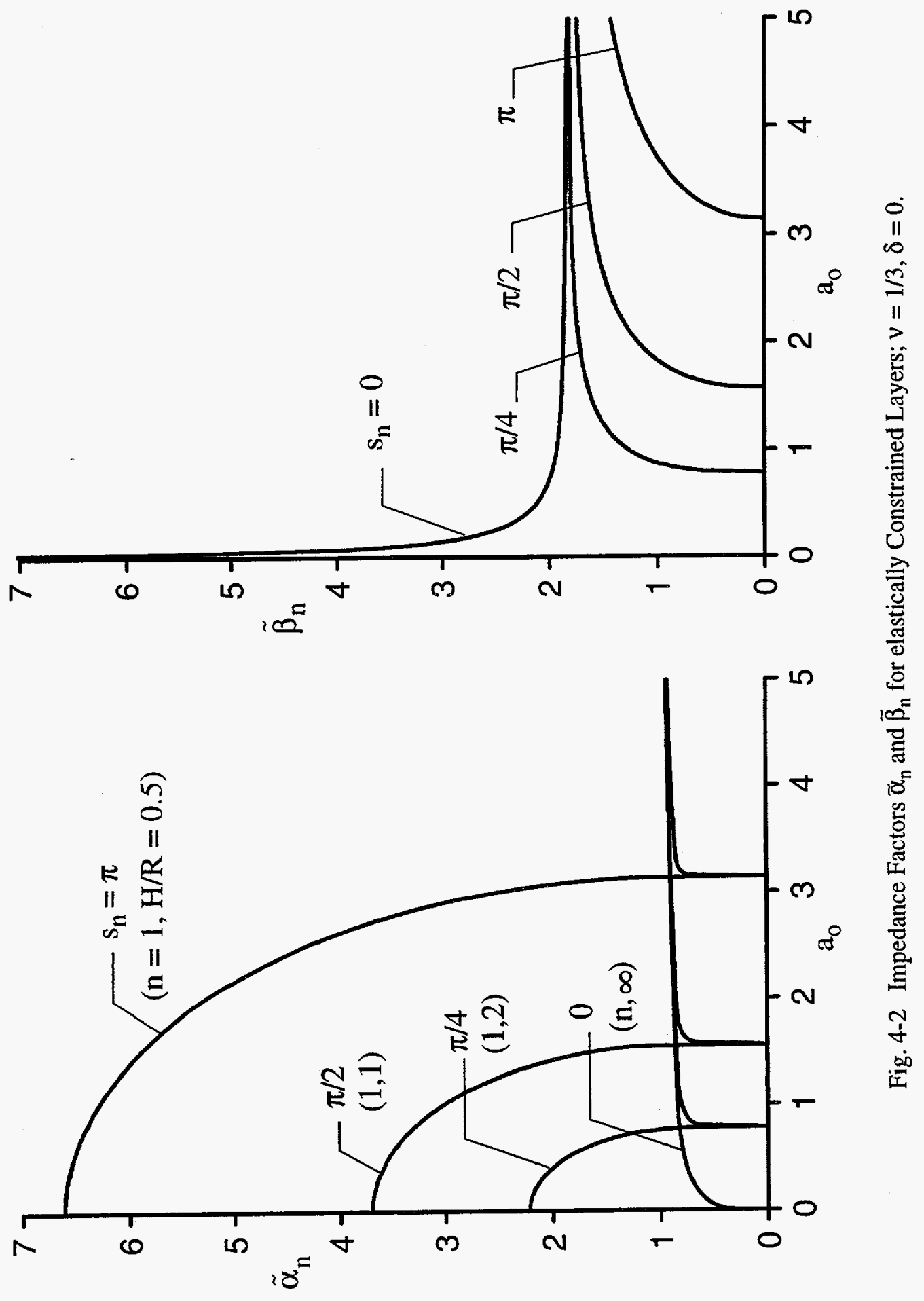




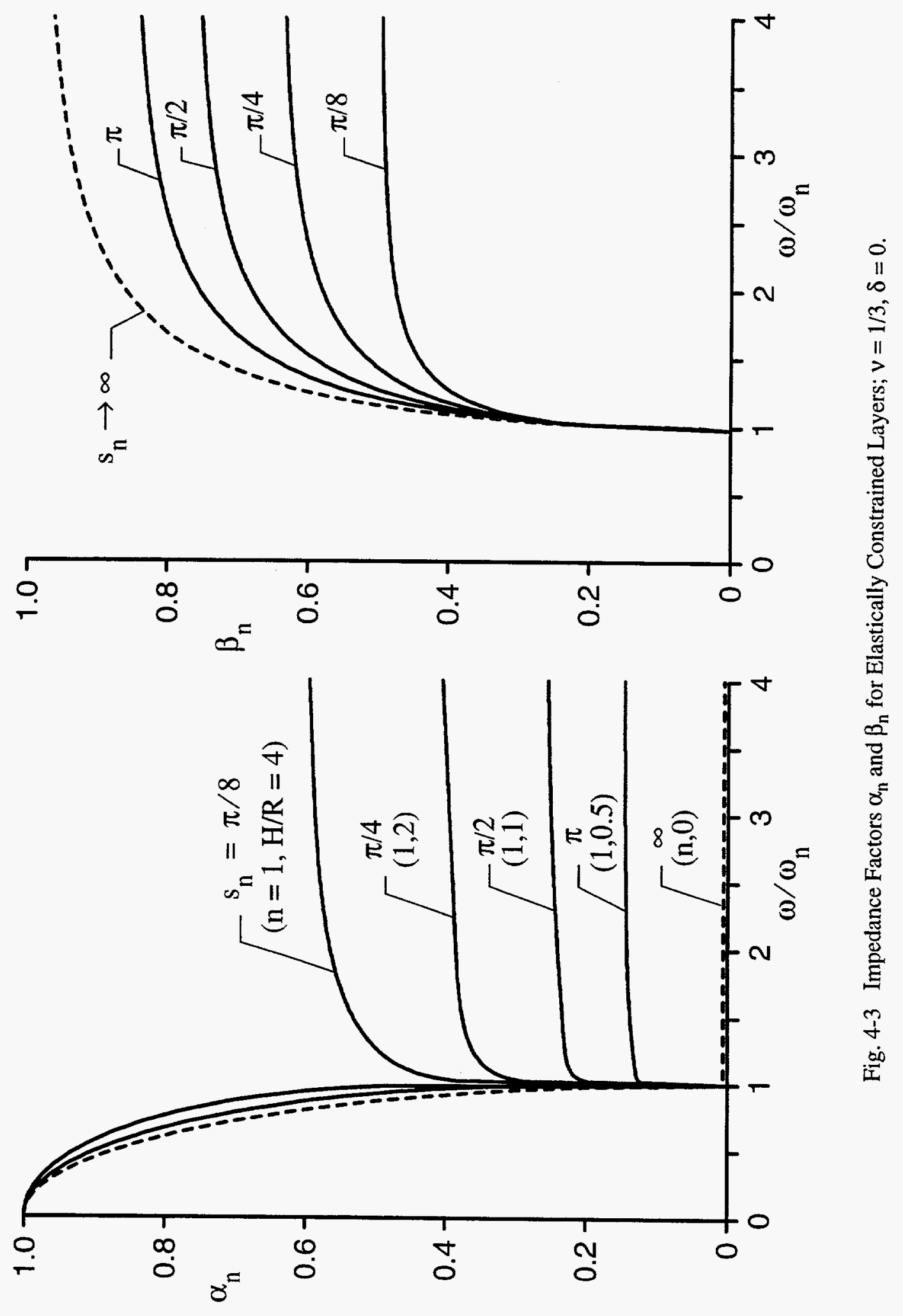


Table 4-1: Values of Dimensionless Coefficient $C_{n}$ in Expression for $\left(K_{n}\right)_{s t}$.

\begin{tabular}{|c|c|c|c|}
\hline $\mathrm{s}_{\mathrm{n}}$ & $v=0$ & $v=1 / 3$ & $v=0.5$ \\
\hline 0 & 0 & 0 & 0 \\
0.1 & 3.258 & 3.638 & 3.881 \\
0.2 & 4.332 & 4.833 & 5.158 \\
0.3 & 5.277 & 5.885 & 6.287 \\
$\pi / 8$ & 6.101 & 6.804 & 7.275 \\
0.4 & 6.165 & 6.875 & 7.352 \\
0.5 & 7.021 & 7.832 & 9.391 \\
0.6 & 7.856 & 8.767 & 9.391 \\
0.7 & 8.677 & 9.687 & 10.387 \\
$\pi / 4$ & 9.371 & 10.465 & 11.229 \\
0.8 & 9.488 & 10.597 & 11.373 \\
0.9 & 10.292 & 11.500 & 12.352 \\
1.0 & 11.089 & 12.397 & 13.326 \\
1.5 & 15.021 & 16.826 & 18.149 \\
$\pi / 2$ & 15.572 & 17.448 & 18.828 \\
2.0 & 18.900 & 21.206 & 22.935 \\
2.5 & 22.753 & 25.561 & 27.702 \\
3.0 & 26.591 & 29.902 & 32.459 \\
$\pi$ & 27.676 & 31.130 & 33.805 \\
5.0 & 41.866 & 47.192 & 51.431 \\
\hline
\end{tabular}




\section{SECTION 5}

\section{RESPONSE DATA FOR CYLINDER-SOIL SYSTEM}

\subsection{Base Shear}

It is desirable to examine first the forces induced on the cylinder by a ground motion the dominant frequencies of which are small compared to the fundamental natural frequency of the stratum (i.e., for motions with $\phi_{1} \rightarrow 0$ ). Such excitations and the resulting effects will be referred to as static, a term which should not be confused with that normally used to represent the effects of gravity forces. The static value of the total horizontal force or base shear for the cylinder, $\left(Q_{b}\right)_{s t}$, is determined from Eq. (31) by letting $\omega=0$ in the expressions for both $K_{n}$ and $U_{n}$. A function of the aspect ratio $H / R$ and of Poisson's ratio for the medium $v$, this force is listed in Table 5-1, normalized with respect to $\pi \rho \ddot{X}_{g} R H^{2}$.

Fig. 5-1 shows the real-valued amplitude of the base shear for the cylinder of harmonically excited systems having four different values of $H / R$ in the range between 0.3 and 10 . The solid lines represent the solutions obtained by the proposed approach using the elastically supported layers, whereas the dashed lines represent those obtained with the unconstrained B-N layers. The results are expressed in the form of amplification factors, which in both cases are normalized with respect to the corresponding static shears listed in Table 5-1, and they are plotted as a function of the frequency ratio $\omega / \omega_{1}$. Poisson's ratio for the medium $v$ and the associated material damping factor $\delta$ in these solutions are taken as $1 / 3$ and 0.10 , respectively. It can be seen that the results obtained with the unconstrained B-N layers may differ significantly from the practically exact results obtained with the constrained layers, the differences being particularly large for the smaller values of $H / R$. The smaller the $H / R$, the greater is the horizontal shearing stiffness of the medium relative to its extensional stiffness, the greater is the proportion of the load transmitted by horizontal shearing action to the base, and, therefore, the less accurate is the modeling of the medium with the conventional, unconstrained B-N layers. For very slender cylinders such as piles, practically the entire load gets transmitted by extensional action, and the conventional B-N approach, as already demonstrated by Nogami and Novak (1977), does yield highly accurate results. This is clearly not the case, however, for the broader systems with relatively small $\mathrm{H} / \mathrm{R}$ values.

The interrelationship of the two solutions sets also depends on the frequency ratio $\omega / \omega_{1}$. At low 
values of $\omega / \omega_{1}$, for which the impedance of the unconstrained $B-N$ layers tends to zero, the conventional approach leads to unacceptably low base shears. By contrast, at high values of $\omega / \omega_{1}$, the two solutions are in a very good agreement; high frequency waves are clearly transmitted by extensional action, and it is immaterial in this case whether the soil layers are constrained or not. The differences between the two solution sets are most pronounced in the intermediate range of frequencies, especially for exciting frequencies that are close to the fundamental natural frequency of the stratum.

Precisely the same trends can also be observed in Fig. 5-2, which defines the amplification factors for base shear in the cylinder induced by the first $6.3 \mathrm{~s}$ of the N-S component of the $1940 \mathrm{El}$ Centro, California earthquake ground motion. The acceleration, velocity and displacement traces of this record are available in Veletsos and Tang (1990), and they are not reproduced here. The peak value of the ground acceleration is $0.312 \mathrm{~g}$, where $\mathrm{g}$ is the gravitational acceleration, and those of the ground velocity and displacement are $35.61 \mathrm{~cm} / \mathrm{s}(14.02 \mathrm{in} / \mathrm{s})$ and $21.05 \mathrm{~cm}(8.29 \mathrm{in})$, respectively. The solid lines define the results obtained by the proposed model with the elastically supported layers, whereas the dashed lines are for the model with the unconstrained B-N layers. Poisson's ratio and the material damping factor for the medium are taken, as before, as $1 / 3$ and 0.1 , respectively. In order that they may easily be compared with those presented in Fig. 5-1, the results are plotted as a function of the fundamental natural period (not frequency) of the medium idealized as a uniform cantilever shearbeam, $T_{1}=2 \pi \omega_{1}$. As a measure of the values of $T_{1}$ that may be encountered in practice, it is noted that for $v_{s}$ in the range between 120 and $500 \mathrm{~m} / \mathrm{s}(394$ and $1640 \mathrm{ft} / \mathrm{s})$ and $\mathrm{H}$ in the range between 6 and $15 \mathrm{~m}$ (19.7 and $49.2 \mathrm{ft}$ ) the value of $\mathrm{T}_{1}$ falls in the range between 0.05 to $0.5 \mathrm{~s}$.

The lower curve in Fig. 5-3 shows the variation with $H / R$ of the average of the four largest amplification factors for base shear obtained by the proposed approach for the El Centro ground motion. Also shown is the corresponding variation of the absolute maximum amplification factor for harmonically excited systems. It is observed that both curves increase with increasing $H / R$, the results for the harmonic excitation being naturally much higher than those for the earthquake ground motion. The larger amplifications for the slender cylinders are due to the reduced capacity of these systems to reflect and to dissipate by radiation the waves that impinge on them.

\subsection{Relative Contributions of Normal and Shearing Stresses}

The forces $F(\eta, t)$ and $Q_{b}(t)$ referred to in the preceding sections are contributed partly by normal stresses, the circumferential variation of which is proportional to $\cos \theta$, and partly by circumferential shearing stress, which vary as $\sin \theta$. If $K_{n}^{\sigma}$ is the component of the layer impedance $K_{n}$ contributed by the normal stresses, and $\mathrm{K}_{\mathrm{n}}^{\tau}$ is the component contributed by the shearing stresses, then $K_{n}=K_{n}^{\sigma}+K_{n}^{\tau}$. The expressions for $K_{n}^{\sigma}$ and $K_{n}^{\tau}$ are identified in the Appendix.

With the values of $K_{n}^{\sigma}$ established, the components of the dynamic base shear and base moment in the 
cylinder contributed by the normal pressures can be evaluated from (31) and (32) merely by replacing $\mathrm{K}_{\mathrm{n}}$ by $\mathrm{K}_{\mathrm{n}}^{\sigma}$.

The real-valued amplitude of the component of the layer impedance contributed by the normal pressures, $\left|K_{n}^{\sigma}\right|$, is plotted in Fig. 5-4 as a function of the frequency ratio $\omega / \omega_{n}$ for four values of the dimensionless stiffness parameter for the supporting springs $s_{n}$. The results are normalized with respect to the real-valued amplitude of the corresponding total impedance, $\left|K_{n}\right|$. Note that, over the complete range of parameters examined, the ratio of the two amplitudes ranges from a low of 0.5 to a high of 0.63. This result is consistent with the conclusion in Veletsos and Younan (1994b) to the effect that, almost irrespective of the system properties and the characteristics of the ground motion, approximately 60 percent of the dynamic base shear in the cylinder is caused by normal pressures, the remaining 40 percent being due to circumferential shearing stresses.

\subsection{Distribution of Cylinder Forces and Base Moment}

It has been shown [Veletsos and Younan (1994b)] that, almost independently of the properties of the medium and the characteristics of ground motion, the fundamental mode of vibration of the unconstrained stratum is the dominant contributor to the response of the system. As a result, the dynamic force per unit of cylinder height may be considered to increase approximately as a half-sine from zero at the base to a maximum value at the top, and the maximum value of the corresponding base moment may be computed as the product of the maximum base shear and a height approximately equal to 60 percent of the stratum thickness. The exact static values of the base moment are listed in Table 5-1.

\subsection{A Final Comment}

The shears and bending moments in the cylinder referred to in the preceding sections represent exclusively the dynamic effects of the normal pressures and circumferential shearing stresses induced by the surrounding medium. To these effects, must also be added the effects of the inertia forces of the cylinder itself. For the rigid cylinder examined, the latter effects may be evaluated readily. 

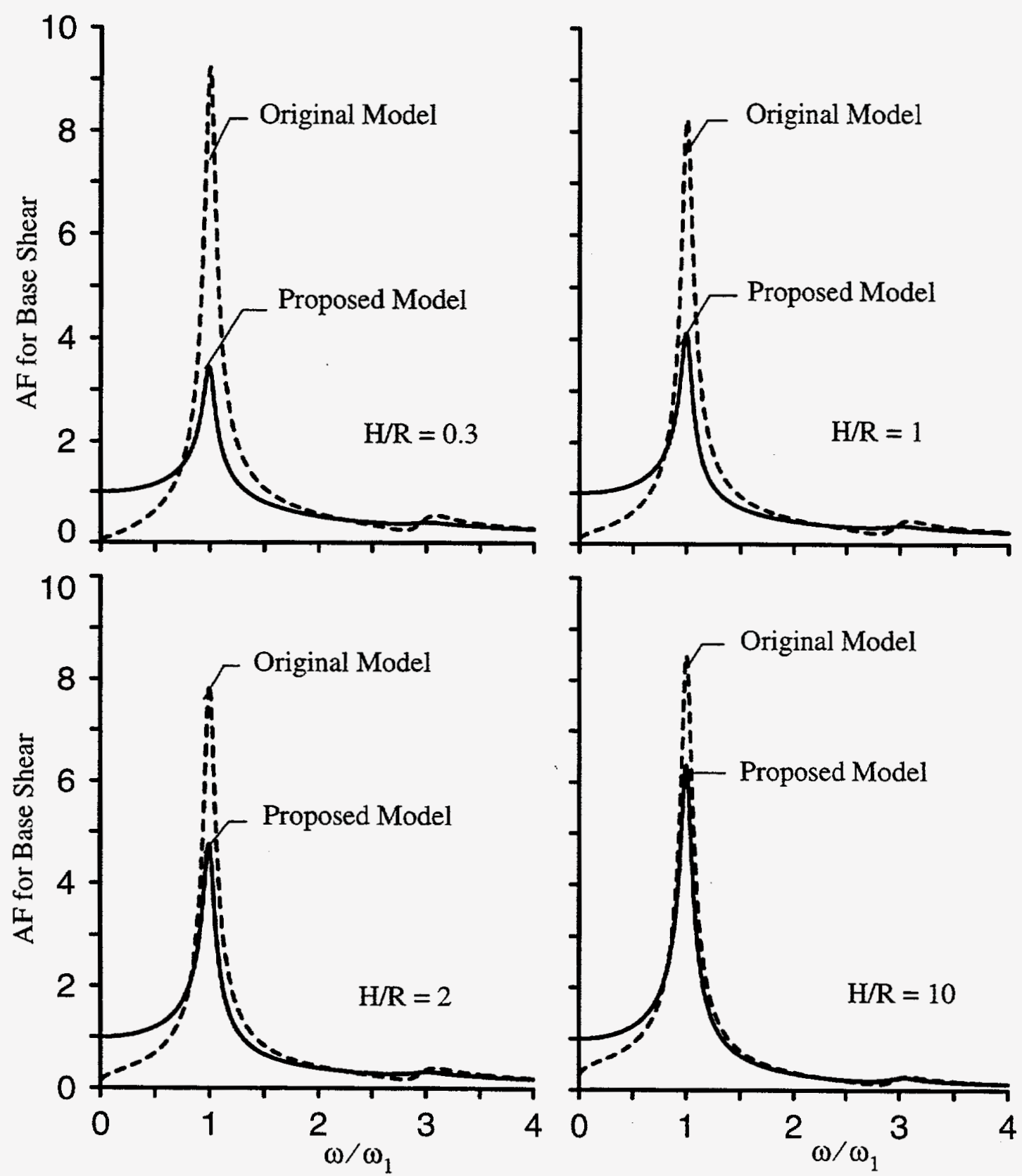

Fig. 5-1 Amplification Factors for Base Shear in Cylinder of Harmonically Excited Systems; $v=1 / 3, \delta=0.1$. 


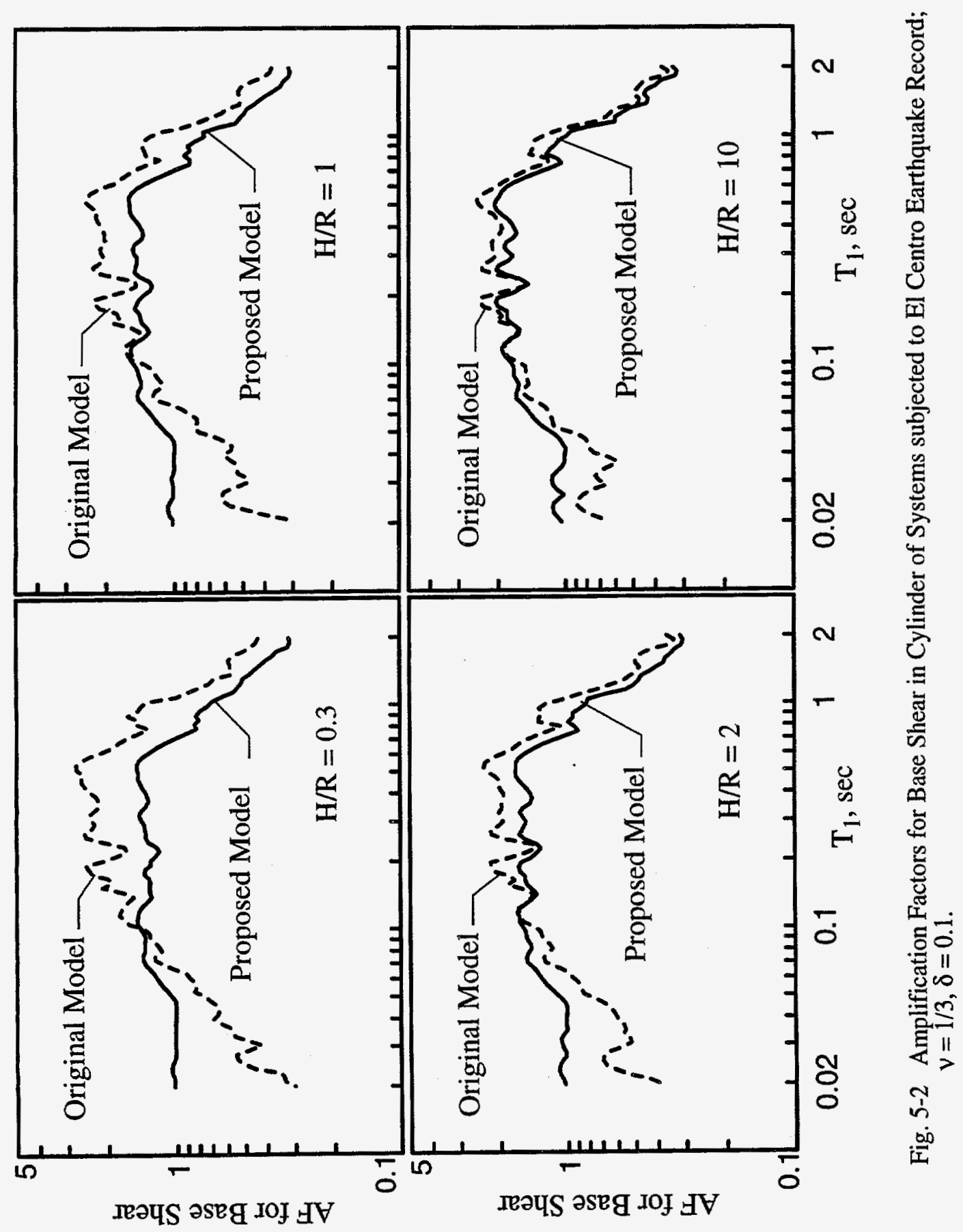




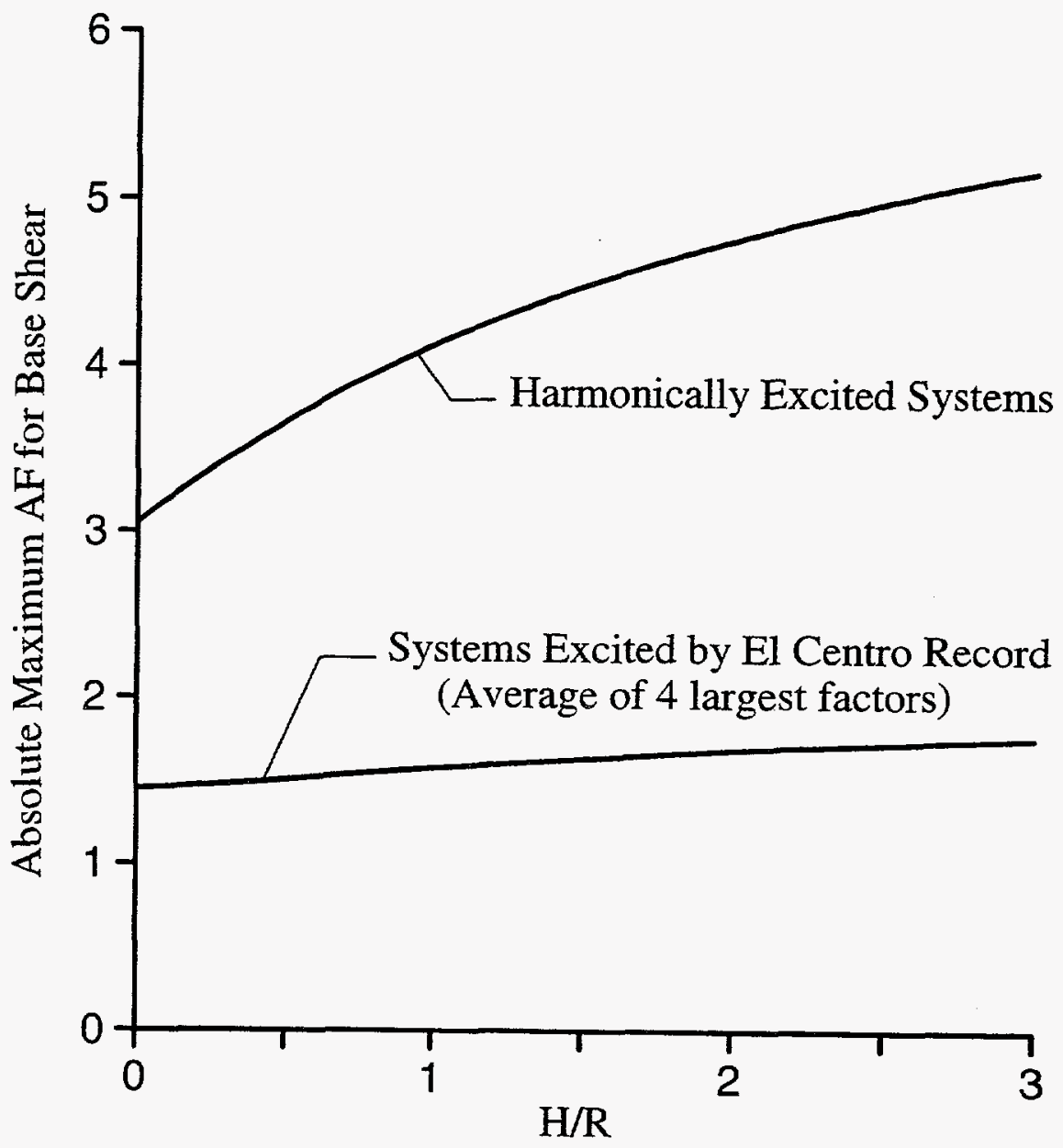

Fig. 5-3 Effect of Stratum-Height to Cylinder-Radius Ratio on Absolute Maximum Amplification Factor for Base Shear in Cylinder of Base-Excited Systems; $v=1 / 3, \delta=0.1$. 


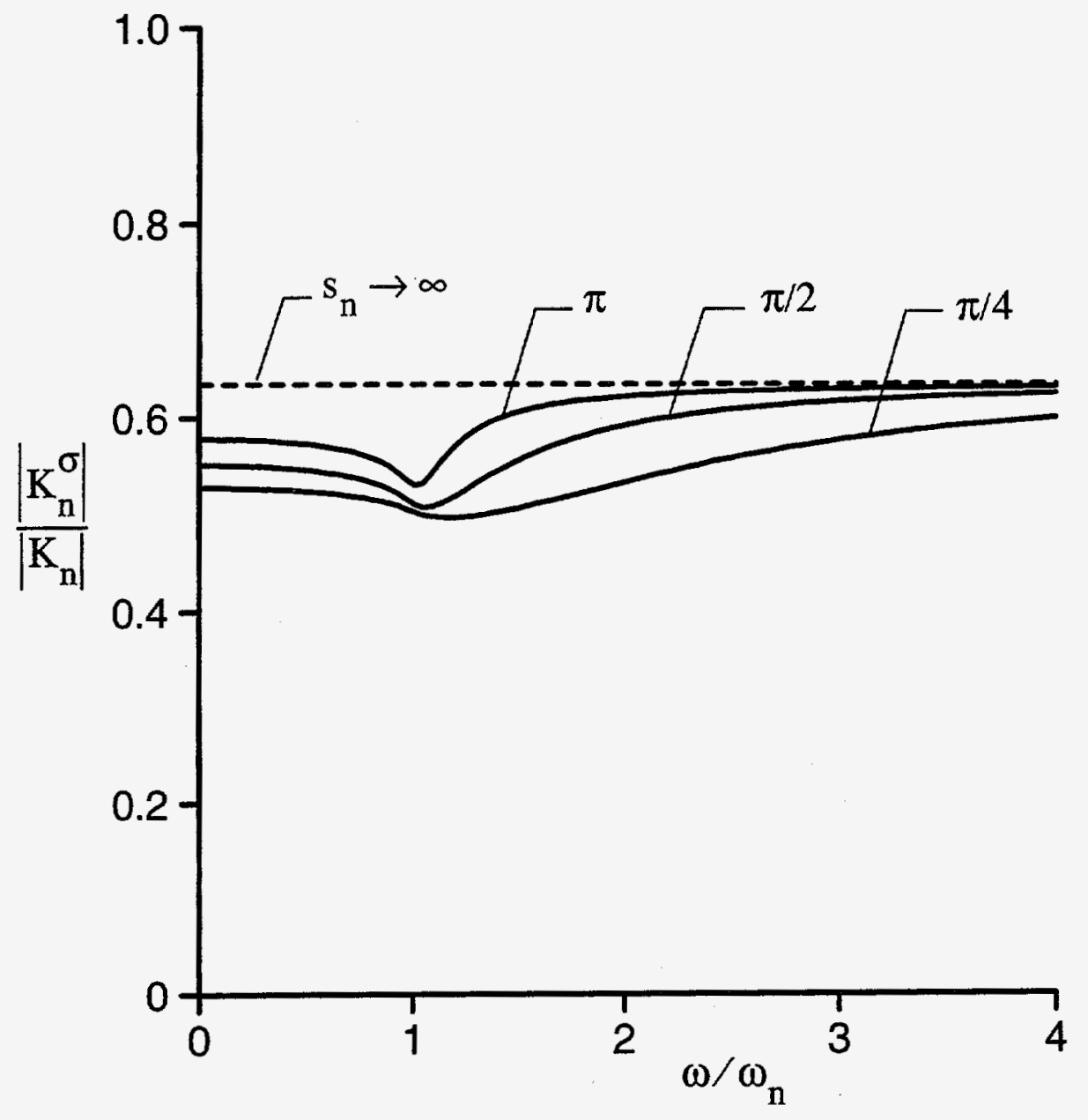

Fig. 5-4 Real-Valued Amplitude of Layer Impedance Contributed by Normal Pressures; $v=1 / 3, \delta=0.1$. 
Table 5-1: Static Values of Base Shear and Base Moment for Systems with Different H/R Ratios.

\begin{tabular}{|c|c|c|c|c|c|c|}
\hline \multirow{2}{*}{$\mathrm{H} / \mathrm{R}$} & \multicolumn{3}{|c|}{$-\frac{\left.\mathrm{Q}_{\mathrm{b}}\right)_{\mathrm{st}}}{\pi \rho \ddot{\mathrm{X}}_{\mathrm{g}} \mathrm{RH}^{2}}$} & \multicolumn{3}{|c|}{$\left.\mathrm{M}_{\mathrm{b}}\right)_{\mathrm{st}}$} \\
\cline { 2 - 7 } & $\mathrm{v}=0$ & $\mathrm{v}=1 / 3$ & $\mathrm{v}=0.5$ & $\mathrm{X}=0$ & $\mathrm{~g}=1 / 3$ & $\mathrm{RH}=0.5$ \\
\hline 0 & 1.310 & 1.482 & 1.627 & 0.784 & 0.888 & 0.975 \\
0.30 & 1.435 & 1.619 & 1.765 & 0.863 & 0.973 & 1.061 \\
0.40 & 1.475 & 1.661 & 1.808 & 0.888 & 1.000 & 1.087 \\
0.50 & 1.514 & 1.703 & 1.850 & 0.912 & 1.026 & 1.114 \\
0.60 & 1.552 & 1.744 & 1.892 & 0.936 & 1.051 & 1.140 \\
0.70 & 1.589 & 1.785 & 1.933 & 0.959 & 1.077 & 1.166 \\
0.80 & 1.626 & 1.825 & 1.974 & 0.982 & 1.102 & 1.191 \\
0.90 & 1.662 & 1.864 & 2.015 & 1.005 & 1.126 & 1.216 \\
1.00 & 1.698 & 1.903 & 2.055 & 1.027 & 1.151 & 1.241 \\
1.25 & 1.786 & 1.999 & 2.154 & 1.081 & 1.210 & 1.303 \\
1.50 & 1.871 & 2.092 & 2.251 & 1.135 & 1.268 & 1.364 \\
1.75 & 1.954 & 2.184 & 2.346 & 1.186 & 1.325 & 1.423 \\
2.00 & 2.035 & 2.273 & 2.440 & 1.237 & 1.381 & 1.482 \\
2.50 & 2.193 & 2.448 & 2.624 & 1.335 & 1.490 & 1.597 \\
3.00 & 2.345 & 2.617 & 2.803 & 1.430 & 1.596 & 1.708 \\
5.00 & 2.917 & 3.253 & 3.476 & 1.786 & 1.992 & 2.128 \\
10.00 & 4.187 & 4.672 & 4.986 & 2.576 & 2.875 & 3.067 \\
\hline
\end{tabular}




\section{SECTION 6}

\section{CONCLUSIONS}

In analyses of the dynamic response of deeply embedded vertical cylindrical structures, the representation of the soil medium by a series of independent Baranov-Novak layers may lead to major inaccuracies, particularly for broad structures with ratios of stratum-height to cylinder-radius of 2 or less. These inaccuracies stem from the failure of this representation to provide for the capacity of the medium between the structure and the far field to transfer forces vertically by horizontal shearing action. The greater the horizontal shearing stiffness of the medium relative to its corresponding extensional stiffness, the greater are generally the resulting discrepancies. This deficiency in modeling may be eliminated by considering the individual layers to be elastically supported to a common base.

In addition to a dimensionless measure of the frequency of the excitation, Poisson's ratio for the medium, and the associated material damping factor, the impedance of the elastically constrained layer depends on the stiffness of the supporting springs. The latter stiffness, in turn, depends on the height-to-radius ratio of the embedded cylinder and on the order of the natural mode in which the soil stratum is vibrating. With the impedances of the elastically constrained layer established, the pressures on and forces in the cylinder for harmonically excited systems may be determined from the expressions presented herein, and those for systems subjected to transient excitations may be evaluated by Fourier transform techniques.

The data presented herein are limited to rigid vertical cylinders and to a non-deformable, moving base. The presence of a flexible rather than rigid cylinder will reduce the effective horizontal extensional stiffness of the medium relative to its shearing stiffness. This reduction, in turn, will increase the forces that get transmitted to the base by horizontal shearing action, and hence the differences between the solutions obtained with the conventional, unconstrained layers and the proposed, constrained layers. By contrast, the presence of a flexible rather than rigid supporting medium will have the opposite effects. These effects will be examined in future publications. 


\section{SECTION 7}

\section{REFERENCES}

Alampalli, S., and Elgamal, A. W. (1991). "Retaining wall; computation of seismically induced deformations." Proc. 2nd Int. Conf. on Recent Adv. in Geotech. Earthquake Eng. and Soil Dyn., St. Louis, Mo., University of Missouri, Rolla, Mo., I, 635-642.

Baranov V.A. (1967). "On the calculation of an embedded foundation," (Russian), Voprosy Dinamiki i Prochnosti, 14, Polytechnical Institute of Riga, Latvia, 195-209.

Beredugo, Y. O., and Novak, M. (1972). "Coupled horizontal and rocking vibration of embedded footings." Canadian Geotech. J., 9 (4), 477-497.

Dennehy, K. T. (1984). "Seismic vulnerability, analysis and design of anchored bulkheads." Ph.D. thesis, Rensselaer Polytechnic Institute, Troy, N.Y.

Gazetas, G., and Dobry, R. (1984), "Simple radiation damping model for piles and footings", J. Eng. Mech. Div., ASCE, 110 (6), 937-956.

Jain, S. K., and Scott, R. F. (1989). "Seismic analysis of cantilever retaining walls." Proc. of Structural Mechanics in Reactor Technology, Anaheim, Calif., 241-246.

Karkanias, S. (1983). "Seismic behavior and simplified analysis of anchored sheet pile bulkheads." M.S. thesis, Rensselaer Polytechnic Institute, Troy, N.Y..

Miller, C. A., Costantino, C. J., and Heymsfeld, E. (1991). "Soil-structure interaction effects on high level waste tanks." Proc. Third D.O.E. Natural Phenomena Hazards Mitigation Conf., St. Louis, Mo., Lawerence Livermore National Laboratory, Livermore, Calif., 588-595.

Nogami, T., and Novak, M. (1977). "Resistance of soil to a horizontally vibrating pile." Earthquake Eng. \& Struct. Dyn., 5 (3), 249-261.

Novak, M. (1974). "Dynamic stiffness and damping of piles." Canadian Geotech. J., 11 (4), 574598.

Novak, M., and Beredugo, Y. O. (1972). "Vertical vibration of embedded footings," J. Soil Mech. and Found. Div., ASCE, 98 (12), 1291-1310.

Novak M., Nogami T. and Aboul-Ella F., (1977) "Dynamic soil reactions for plane strain case," Research Report BLWT-1-77, Faculty of Engineering Science, The University of Western Ontario, London, Ontario, Canada, 1-26. 
Novak, M., Nogami T. and Aboul-Ella F., (1978) "Dynamic soil reactions for plane strain case," $J$. Eng. Mech. Div., ASCE, 104 (4), 953-959.

Novak M., and Sachs, K. (1973), "Torsional and coupled vibrations of embedded footings," Earthquake Eng. \& Struct. Dyn., 2 (1), 11-33.

Pais, A., and Kausel, E. (1988). "Approximate formulas for dynamic stiffnesses of rigid foundations." Soil Dyn. \& Earthquake Eng., 7 (4), 213-227.

Roesset, J. M., Whitman, R. V., and Dobry, R. (1973). "Modal analysis for structures with foundation interaction." J. Struct. Div., ASCE, 99 (3), 399-416.

Scott, R. F. (1973). "Earthquake-induced pressures on retaining walls." Proc. 5th World Conf. on Earthquake Eng., Rome, Italy, LAEE, Tokyo, Japan, II, 1611-1620.

Sokolnikoff, I. S. (1983). Mathematical theory of elasticity, R. E. Krieger Co., Malabar, Florida, 253254.

Tajimi, H. (1969), "Dynamic Analysis of a Structure Embedded in an Elastic Stratum," Proc. of 4th World Conf. on Earthquake Eng., San Diego, Chile, IAEE, Tokyo, Japan, III(A-6), 53-69.

Tassoulas, J. L., and Kausel, E. (1983). "On the effects of the rigid sidewall on the dynamic stiffness of embedded circular footings." Earthquake Eng. \& Struct. Dyn., 11 (3), 403-414.

Veletsos, A. S., and Dotson, K. W., (1988), "Horizontal impedances for radially inhomogeneous viscoelastic soil layers," Earthquake Eng. \& Struct. Dyn., 16 (7), 947-966.

Veletsos, A. S., and Tang, Y. (1990). "Deterministic assessment of effects of ground-motion incoherence." J. Eng. Mech. Div., ASCE, 116(5), 1109-1124.

Veletsos, A. S., and Verbic, B. (1973). "Vibration of viscoelastic foundations." Earthquake Eng. \& Struct. Dyn., 2 (1), 87-102.

Veletsos, A. S., and Younan, A. H. (1994a). "Dynamic soil pressures on rigid vertical walls." Earthquake Eng. \& Struct. Dyn., 23 (3), 275-301; also available as Report 52357, Brookhaven National Laboratory, Upton, N.Y

Veletsos, A. S., and Younan, A. H. (1994b). "Dynamic soil pressures on rigid cylindrical vaults." Earthquake Eng. \& Struct. Dyn., 23 (6), 645-669; also available as Report 52372, Brookhaven National Laboratory, Upton, N.Y.

Veletsos A. S. and Younan A. H. (1994c), "Dynamic modeling and response of soil-wall systems," to appear in J. Geotech. Eng. Div., ASCE, Paper 7061, December 1994; also available as Report 52402, Brookhaven National Laboratory, Upton, N.Y. 


\section{SECTION 8}

\section{APPENDIX}

The equations of motion of the elastically constrained layer in the radial and circumferential directions are

$$
\begin{aligned}
& \frac{1}{R}\left[\frac{\partial \sigma_{\mathrm{r}}}{\partial \xi}+\frac{1}{\xi} \frac{\partial \tau_{\mathrm{r} \theta}}{\partial \theta}+\frac{\sigma_{\mathrm{r}}-\sigma_{\theta}}{\xi}\right]=\rho \frac{\partial^{2} \mathrm{u}}{\partial \mathrm{t}^{2}}+k_{\mathrm{n}}^{*} \mathrm{u} \\
& \frac{1}{\mathrm{R}}\left[\frac{\partial \tau_{\mathrm{r} \theta}}{\partial \xi}+\frac{1}{\xi} \frac{\partial \sigma_{\theta}}{\partial \theta}+\frac{2}{\xi} \tau_{\mathrm{r} \theta}\right]=\rho \frac{\partial^{2} \mathrm{v}}{\partial \mathrm{t}^{2}}+k_{\mathrm{n}}^{*} \mathrm{v}
\end{aligned}
$$

where $\sigma_{\mathrm{r}}$ and $\sigma_{\theta}$ are the radial and circumferential normal stresses; $\tau_{\mathrm{r} \theta}$ is the shearing stress in the r$\theta$ plane; and all other symbols are as previously defined.

For the plane stress condition considered, the stress components are related to the displacement components $\mathrm{u}$ and $\mathrm{v}$ by [Sokolnikoff (1983)]

$$
\begin{aligned}
& \sigma_{\mathrm{r}}=\frac{2 \Lambda^{*} \mathrm{G}^{*}}{\Lambda^{*}+2 \mathrm{G}^{*}}\left[\frac{\partial \mathrm{u}}{\partial \xi}+\frac{\mathrm{u}}{\xi}+\frac{1}{\xi} \frac{\partial \mathrm{v}}{\partial \theta}\right]+\frac{2 \mathrm{G}^{*}}{\mathrm{R}} \frac{\partial \mathrm{u}}{\partial \xi} \\
& \sigma_{\theta}=\frac{2 \Lambda^{*} \mathrm{G}^{*}}{\Lambda^{*}+2 \mathrm{G}^{*}}\left[\frac{\partial \mathrm{u}}{\partial \xi}+\frac{\mathrm{u}}{\xi}+\frac{1}{\xi} \frac{\partial \mathrm{v}}{\partial \theta}\right]+\frac{2 \mathrm{G}^{*}}{\mathrm{R}}\left[\frac{\mathrm{u}}{\xi}+\frac{1}{\xi} \frac{\partial \mathrm{v}}{\partial \theta}\right] \\
& \tau_{\mathrm{r} \theta}=\frac{\mathrm{G}^{*}}{\mathrm{R}}\left[\frac{\partial \mathrm{v}}{\partial \xi}-\frac{\mathrm{v}}{\xi}+\frac{1}{\xi} \frac{\partial \mathrm{u}}{\partial \theta}\right]
\end{aligned}
$$

where $\mathrm{G}^{*}$ is the complex-valued shear modulus defined by (21) and

$$
\Lambda^{*}=\frac{2 v}{1-2 v} G^{*}
$$

The remaining steps in the following development are similar to those followed by Novak et al. (1977), (1978), and by Veletsos and Dotson (1988) in their evaluation of the impedance of the unconstrained B-N layers. Specifically, the displacements $u$ and $v$ are expressed in terms of the potential functions $\phi$ and $\psi$ as 


$$
\begin{aligned}
& \mathrm{u}=\frac{\partial \phi}{\partial \xi}+\frac{1}{\xi} \frac{\partial \psi}{\partial \theta} \\
& \mathrm{v}=\frac{1}{\xi} \frac{\partial \phi}{\partial \theta}-\frac{\partial \psi}{\partial \xi}
\end{aligned}
$$

and use is made of Eqs. (37) through (39) to rewrite Eqs. (35) and (36) as

$$
\begin{aligned}
& \psi_{0}^{2} \frac{\mathrm{G}^{*}}{\mathrm{R}^{2}}\left[\frac{\partial^{2} \phi}{\partial \xi^{2}}+\frac{1}{\xi} \frac{\partial \phi}{\partial \xi}+\frac{1}{\xi^{2}} \frac{\partial^{2} \phi}{\partial \theta^{2}}\right]=\rho \frac{\partial^{2} \phi}{\partial \mathrm{t}^{2}}+\mathrm{k}_{\mathrm{n}}^{*} \phi \\
& \frac{\mathrm{G}^{*}}{\mathrm{R}^{2}}\left[\frac{\partial^{2} \psi}{\partial \xi^{2}}+\frac{1}{\xi} \frac{\partial \psi}{\partial \xi}+\frac{1}{\xi^{2}} \frac{\partial^{2} \psi}{\partial \theta^{2}}\right]=\rho \frac{\partial^{2} \psi}{\partial \mathrm{t}^{2}}+\mathrm{k}_{\mathrm{n}}^{*} \psi
\end{aligned}
$$

where $\psi_{0}$ is a dimensionless function of Poisson's ratio, defined by

$$
\Psi_{0}=\sqrt{2+\frac{2 \Lambda^{*}}{\Lambda^{*}+2 G^{*}}}=\sqrt{\frac{2}{1-v}}
$$

For the harmonically excited layer,

$$
\begin{aligned}
& \phi=\Phi(\xi) \cos \theta \mathrm{e}^{\mathrm{i} \omega t} \\
& \Psi=\Psi(\xi) \sin \theta \mathrm{e}^{i \omega t} \\
& \mathbf{u}=\mathrm{U}(\xi) \cos \theta \mathrm{e}^{\mathrm{i} \omega t} \\
& \mathrm{v}=\mathrm{V}(\xi) \sin \theta \mathrm{e}^{\mathrm{i} \omega \mathrm{t}} \\
& \sigma_{\mathrm{r}}=\sigma(\xi) \cos \theta \mathrm{e}^{\mathrm{i} \omega t} \\
& \tau_{\mathrm{r} \theta}=\tau(\xi) \sin \theta \mathrm{e}^{\mathrm{i} \omega t}
\end{aligned}
$$

and Eqs. (43) and (44) reduce to the system of ordinary differential equations

$$
\begin{aligned}
& \frac{\mathrm{d}^{2} \Phi}{\mathrm{d}^{2}}+\frac{1}{\xi} \frac{\mathrm{d} \Phi}{\mathrm{d} \xi}-\left[\mu^{2}+\frac{1}{\xi^{2}}\right] \Phi=0 \\
& \frac{\mathrm{d}^{2} \Psi}{\mathrm{d}^{2}}+\frac{1}{\xi} \frac{\mathrm{d} \Psi}{\mathrm{d} \xi}-\left[\lambda^{2}+\frac{1}{\xi^{2}}\right] \Psi=0
\end{aligned}
$$

where 


$$
\begin{aligned}
& \lambda=\sqrt{\mathrm{s}_{\mathrm{n}}^{2}-\frac{\mathrm{a}_{0}^{2}}{1+\mathrm{i} \delta}} \\
& \mu=\frac{\lambda}{\Psi_{0}}
\end{aligned}
$$

$s_{n}$ is the dimensionless stiffness parameter for the supporting springs defined by Eq. (24); and $a_{0}$ is the dimensionless frequency parameter defined by Eq. (2).

On making use of the fact that the layer is at rest as $r \rightarrow \infty$, the solution of Eqs. (52) and (53) can be written as

$$
\begin{aligned}
& \Phi(\xi)=\mathrm{AK}_{1}(\mu \xi) \\
& \Psi(\xi)=\mathrm{B} \mathrm{K}_{1}(\lambda \xi)
\end{aligned}
$$

where $K_{1}$, not to be confused with the impedance $K_{n}$, is the modified Bessel function of the first order and second kind, and $A$ and $B$ are constants of integration that must be determined from the conditions along the inner circular boundary. For the fully bonded inner boundary considered, the latter conditions are given by

$$
\begin{aligned}
& U(1)=1 \\
& V(1)=-1
\end{aligned}
$$

On substituting Eqs. (56) and (57) along with Eqs. (46) through (49), into Eqs. (41) and (42), the following expressions are obtained for the complex-valued displacement amplitudes

$$
\begin{aligned}
& \mathrm{U}(\xi)=-\mathrm{A}\left[\frac{1}{\xi} \mathrm{K}_{1}(\mu \xi)+\mu \mathrm{K}_{0}(\mu \xi)\right]+\mathrm{B} \frac{1}{\xi} \mathrm{K}_{1}(\lambda \xi) \\
& \mathrm{V}(\xi)=-\mathrm{A} \frac{1}{\xi} \mathrm{K}_{1}(\mu \xi)+\mathrm{B}\left[\frac{1}{\xi} \mathrm{K}_{1}(\lambda \xi)+\lambda \mathrm{K}_{0}(\lambda \xi)\right]
\end{aligned}
$$

where the subscripts 0 and 1 on $\mathrm{K}$ denote the order of the Bessel function, and the integration constants A and B are determined from Eqs. (58) and (59) to be

$$
\begin{aligned}
& A=-\frac{1}{\Delta}\left[2 K_{1}(\lambda)+\lambda K_{0}(\lambda)\right] \\
& B=-\frac{1}{\Delta}\left[2 K_{1}(\mu)+\mu K_{0}(\mu)\right]
\end{aligned}
$$

with

$$
\Delta=\left[\mathrm{K}_{1}(\mu)+\mu \mathrm{K}_{0}(\mu)\right]\left[\mathrm{K}_{1}(\lambda)+\lambda \mathrm{K}_{0}(\lambda)\right]-\mathrm{K}_{1}(\mu) \mathrm{K}_{1}(\lambda)
$$


The complex-valued amplitudes of the normal stresses and circumferential shearing stresses along the inner circular boundary, $\sigma(1)$ and $\tau(1)$, are then determined from Eqs. (37) and (39) to be

$$
\begin{aligned}
& \sigma(1)=\psi_{0}^{2} \frac{\mathrm{G}^{*}}{\mathrm{R}}\left[\mathrm{A}\left\{\left(2+\mu^{2}\right) \mathrm{K}_{1}(\mu)+\mu \mathrm{K}_{0}(\mu)\right\}-\mathrm{B}\left\{2 \mathrm{~K}_{1}(\lambda)+\lambda \mathrm{K}_{0}(\lambda)\right\}\right] \\
& \tau(1)=\frac{\mathrm{G}^{*}}{\mathrm{R}}\left[\mathrm{A}\left\{2 \mathrm{~K}_{1}(\mu)+\mu \mathrm{K}_{0}(\mu)\right\}-\mathrm{B}\left\{\left(2+\lambda^{2}\right) \mathrm{K}_{1}(\lambda)+\lambda \mathrm{K}_{0}(\lambda)\right\}\right]
\end{aligned}
$$

The impedance $\mathrm{K}_{\mathrm{n}}$ of the elastically constrained layer is finally determined by integration of these stresses as

$$
\mathrm{K}_{\mathrm{n}}=-\int_{0}^{2 \pi}\left\{\sigma(1) \cos ^{2} \theta-\tau(1) \sin ^{2} \theta\right\} \mathrm{Rd} \theta=-\pi \mathrm{R}[\sigma(1)-\tau(1)]
$$

The real part of $K_{n}$ represents the product $1.5 \pi \tilde{\alpha}_{n} G$ in (23), and the imaginary part represents the product $1.5 \pi \mathrm{a}_{0} \tilde{\beta}_{\mathrm{n}} \mathrm{G}$. The static stiffness of the layer, $\left(\mathrm{K}_{\mathrm{n}}\right)_{\mathrm{s}}$, is the value of $\mathrm{K}_{\mathrm{n}}$ corresponding to $a_{o}=0$. Finally, the impedances for the unconstrained layer displayed in Fig. 2(b) are obtained from (67) by letting $s_{n}=0$ in the expressions for $\sigma(1)$ and $\tau(1)$.

The part of $\mathrm{K}_{\mathrm{n}}$ contributed by the normal pressures, $\mathrm{K}_{\mathrm{n}}^{\sigma}$, is given by

$$
\mathrm{K}_{\mathrm{n}}^{\sigma}=-\pi \mathrm{R} \sigma(1)
$$

and the part contributed by the shearing stresses, $K_{n}^{\tau}$, is given by

$$
\mathrm{K}_{\mathrm{n}}^{\tau}=\pi \mathrm{R} \tau(1)
$$

As $s_{n} \rightarrow \infty, K_{n}^{\sigma}$ divided by $\pi R$ can be shown to reduce, as it should, to the impedance of a semiinfinite, uniform bar of unit cross sectional area. The latter stiffness is defined by equation (23) in Veletsos and Younan (1994c). 


\section{SECTION 9}

\section{NOTATION}

The following symbols are used in this study:

$a_{0} \quad$ dimensionless frequency parameter defined by Eq. (2);

$C_{n}$ dimensionless function in expression for $\left(K_{n}\right)_{s t}$;

$F \quad$ force per unit of cylinder height;

G shear modulus of elasticity of soil material;

G* complex valued shear modulus;

$\mathrm{g}_{\mathrm{n}} \quad$ function in modal expansion of $\mathrm{u}$;

$\mathrm{H}$ height of cylinder and soil stratum;

$h_{n} \quad$ function in modal expansion of $v$;

i $\quad \sqrt{-1}$;

$\mathrm{K}$ impedance of unconstrained layer;

$\mathrm{K}_{\mathrm{n}} \quad$ impedance of elastically supported layer when vibrating in nth natural mode of stratum;

$\mathrm{K}_{\mathrm{n}}^{\sigma} \quad$ component of $\mathrm{K}_{\mathrm{n}}$ contributed by normal stresses;

$\mathrm{K}_{\mathrm{n}}^{\tau} \quad$ component of $\mathrm{K}_{\mathrm{n}}$ contributed by shearing stresses;

$\left(K_{n}\right)_{\text {st }}$ static value of $K_{n}$;

$\mathrm{k}_{\mathrm{n}}$

stiffness of springs supporting thin soil layer when the stratum is vibrating in its nth natural mode;

$\mathrm{k}_{\mathrm{n}}^{*} \quad$ complex valued stiffness of supporting springs;

$\mathrm{M}_{\mathrm{b}} \quad$ bending moment at cylinder base;

$\mathrm{n} \quad$ positive integer;

$\mathrm{Q}_{\mathrm{b}} \quad$ base shear in cylinder;

$\left(Q_{b}\right)_{s t} \quad$ static value of base shear;

$\mathrm{r}$ radial position coordinate;

$\mathrm{R} \quad$ radius of cylinder;

$T_{1} \quad$ fundamental natural period of soil stratum;

t time;

u radial component of displacement of medium relative to moving base;

$u_{f} \quad$ horizontal displacement relative to the moving base of a point of the stratum at far-field;

$u_{n} \quad$ nth modal component of $u$;

$\mathrm{U}_{\mathrm{f}} \quad$ amplitude of $\mathrm{u}_{\mathrm{f}}$; 
$U_{n} \quad n$th term in an expansion of $U_{f}$ in terms of its modal components;

$\mathrm{v} \quad$ circumferential component of displacement;

$\mathrm{v}_{\mathrm{c}} \quad$ velocity of compressional wave in medium;

$v_{c}^{\prime} \quad$ wave velocity defined by Eq. (34);

$\mathrm{v}_{\mathrm{n}} \quad$ nth modal component of $\mathrm{v}$;

$v_{s} \quad$ velocity of shear-wave wave in medium;

$s_{\mathrm{n}} \quad$ dimensionless measure of stiffness $\mathrm{k}_{\mathrm{n}}$;

$\ddot{X}_{\mathrm{g}} \quad$ maximum value of ground acceleration;

$\ddot{x}_{g}(t)$ instantaneous value of ground acceleration;

$\mathrm{z} \quad$ vertical position coordinate;

$\alpha \quad$ stiffness coefficient in expression for $\mathrm{K}$;

$\alpha_{n} \quad$ stiffness coefficient in Eq. (23) for the layer impedance $K_{n}$;

$\tilde{\alpha}_{n} \quad$ stiffness coefficient in Eq. (28) for the layer impedance $K_{n}$;

$\beta \quad$ damping coefficient in expression for $\mathrm{K}$;

$\beta_{n} \quad$ damping coefficient in Eq. (23) for the layer impedance $K_{n}$;

$\tilde{\beta}_{\mathrm{n}}$ damping coefficient in Eq. (28) for the layer impedance $K_{n}$;

$\Delta \tau_{\mathrm{zr}} \quad$ difference in radial component of shearing stresses;

$\Delta \tau_{z \theta}$ difference in circumferential component of shearing stresses;

$\left(\Delta \tau_{\mathrm{zr}}\right)_{\mathrm{r}}$ nth modal component of $\Delta \tau_{\mathrm{zr}}$;

$\left(\Delta \tau_{z \theta}\right)_{\mathrm{n}} \quad$ nth modal component of $\Delta \tau_{z \theta}$;

$\delta \quad$ material damping factor for soil layer;

$\eta \quad z / H=$ dimensionless vertical position coordinate;

$\theta$ angle measured counterclockwise from an axis parallel to the direction of the applied ground excitation;

$v \quad$ Poisson's ratio for material of stratum;

$\xi \quad \mathrm{r} / \mathrm{H}=$ dimensionless radial position coordinate;

$\rho \quad$ mass density for material of stratum;

$\tau_{\mathrm{zr}} \quad$ radial shearing stress;

$\tau_{z \theta} \quad$ circumferential shearing stress;

$\phi_{\mathrm{n}} \quad \omega / \omega_{\mathrm{n}}$ frequency ratio defined by Eq. (7);

$\psi_{0} \quad$ dimensionless function of Poisson's ratio defined by Eq. (45) in Appendix;

$\omega \quad$ circular frequency of excitation and of resulting steady-state response;

$\omega_{n} \quad n$th circular natural frequency of stratum considered to act as a series of vertical cantilever shear-beams. 